\title{
Practical acquisition and rendering of diffraction effects in surface reflectance
}

\author{
ANTOINE TOISOUL, Imperial College London \\ ABHIJEET GHOSH, Imperial College London
}

\begin{abstract}
We propose two novel contributions for measurement based rendering of diffraction effects in surface reflectance of planar homogeneous diffractive materials. As a general solution for commonly manufactured materials, we propose a practical data-driven rendering technique and a measurement approach to efficiently render complex diffraction effects in real-time. Our measurement step simply involves photographing a planar diffractive sample illuminated with an LED flash. Here, we directly record the resultant diffraction pattern on the sample surface due to a narrow band point source illumination. Furthermore, we propose an efficient rendering method that exploits the measurement in conjunction with the Huygens-Fresnel principle to fit relevant diffraction parameters based on a first order approximation. Our proposed data-driven rendering method requires the precomputation of a single diffraction look up table for accurate spectral rendering of complex diffraction effects. Secondly, for sharp specular samples, we propose a novel method for practical measurement of the underlying diffraction grating using out-of-focus "bokeh" photography of the specular highlight. We demonstrate how the measured bokeh can be employed as a height field to drive a diffraction shader based on a first order approximation for efficient real-time rendering. Finally, we also drive analytic solutions for a few special cases of diffraction from our measurements and demonstrate realistic rendering results under complex light sources and environments.
\end{abstract}

CCS Concepts: • Computing methodologies $\rightarrow$ Reflectance modeling; Computational photography;

Additional Key Words and Phrases: diffraction, reflectance, acquisition, spectral, real-time rendering

ACM Reference format:

Antoine Toisoul and Abhijeet Ghosh. 2017. Practical acquisition and rendering of diffraction effects in surface reflectance. ACM Trans. Graph. 36, N, Article xx (September 2017), 16 pages.

https://doi.org/http://dx.doi.org/10.1145/3012001

\section{INTRODUCTION}

Appearance modeling of real world materials has received significant attention over the years in computer graphics for realistic rendering applications as well as in computer vision for material recognition and classification applications. However, much of the effort has been focused on measurement and modeling of everyday materials where the surface reflectance of incident light is well explained by standard geometric optics. This is because geometric optics is valid for modeling surface reflectance in a vast majority of materials spanning dielectrics and metals, and can effectively model both isotropic [Marschner et al. 1999; Matusik et al. 2003] and anisotropic [Ngan et al. 2005; Wang et al. 2008; Ward 1992] surface reflectance. However, geometric optics based reflectance models

Authors' address: Dept. of Computing, Imperial College London, South Kensington Campus SW7 2RH, UK.

(c) 2017 Association for Computing Machinery.

This is the author's version of the work. It is posted here for your personal use. Not for redistribution. The definitive Version of Record was published in ACM Transactions on Graphics, https://doi.org/http://dx.doi.org/10.1145/3012001.

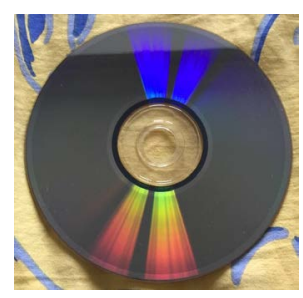

(a)

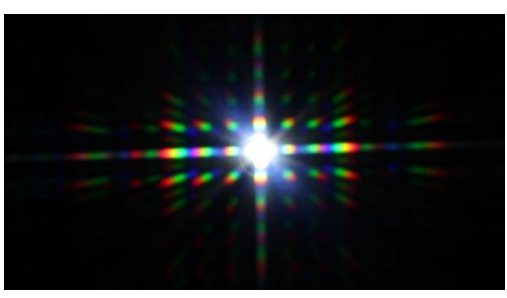

(b)
Fig. 1. (a) Diffraction observed on a compact disc. (b) Diffraction pattern produced by a LCD phone screen.

are unable to model iridescence due to wave effects in surface reflectance which are characteristic of certain materials. Iridescences can produce impressive visual effects and interesting color patterns in surface reflectance and are primarily caused by diffraction or thin film interference, although in some cases they can also be caused by dispersive refraction or scattering [Hecht 2014].

In this work, we focus on iridescence due to diffraction effects in surface reflectance which are becoming increasingly common in our everyday life. Such effects can be observed in modern manufactured materials such as optical discs, LCD screens, holographic paper, etc. (see Figs. 1 and 2), as well as in certain biological structures. In such materials, the visible iridescence is due to diffraction of light which happens when the microgeometry of the surface reaches a size close to the wavelength of light (around $0.5 \mu \mathrm{m}$ ). For instance, on a compact disc (CD) the bits of information are stored along tracks that are separated by a distance of around $2.0 \mu \mathrm{m}$ which produce the separation of white light (Fig. 1, a). In modern LCD screens, strong diffraction patterns can be observed (Fig. 1, b) due to the high density of pixels on high definition screens where individual pixels now reach a size $(16-25 \mu \mathrm{m})$ which is below the coherence length of white light $(65 \mu \mathrm{m})$ [Mashaal et al. 2012]. Such surface microstructure is often called a diffraction grating for periodic structures which are characteristic of manufactured materials.

Optically, such diffraction is the result of reflection of light waves on a diffraction grating which produces interference between waves. For a given wavelength, the resulting intensity is zero for destructive interferences (sum of out of phase waves) and doubled for constructive interferences (sum of waves in phase). The constructive and destructive interferences happen at different angles depending on the wavelength of light. Hence, white light is separated into its main colours producing colourful patterns. The colours always happen in the same order from violet to red (short wavelengths to longer wavelengths) and repeat spatially with each repetition of the spectral decomposition referred to as an order of diffraction (Fig. 3).

The rendering of diffraction effects has traditionally been somewhat challenging in computer graphics due to the computational 


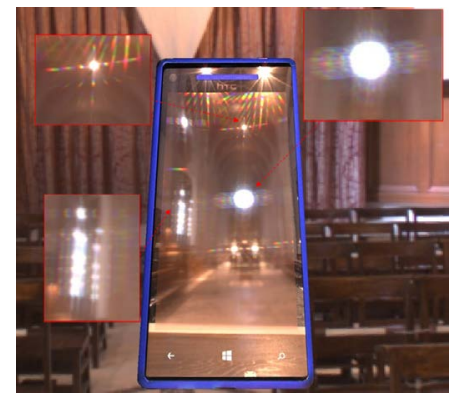

(a)

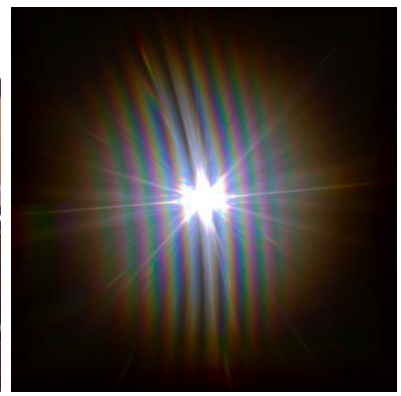

(b)

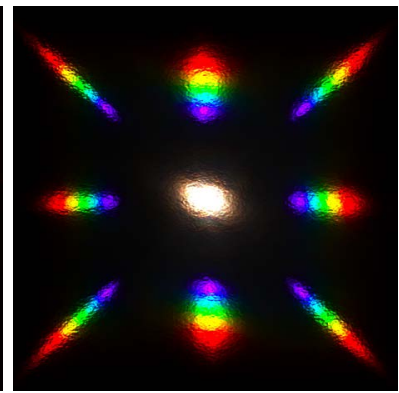

(c)
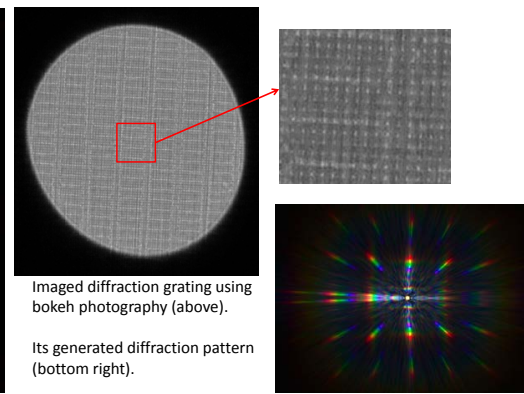

(d)

Fig. 2. Renderings of diffraction effects observed in surface reflectance of a few common materials. (a) - (c) Proposed data-driven rendering results. (a) Diffraction patterns produced by an HTC 8 X phone screen due to complex environmental illumination. (b, c) Diffraction patterns due to a point light source: (b) Bragg diffraction produced by a laptop (Lenovo Yoga) LCD screen, and (c) diffraction on a holographic paper. (d) Diffraction grating of an LG G3 phone screen measured using proposed "bokeh" photography and the resulting diffraction pattern rendered using a first order approximation (bottom right).

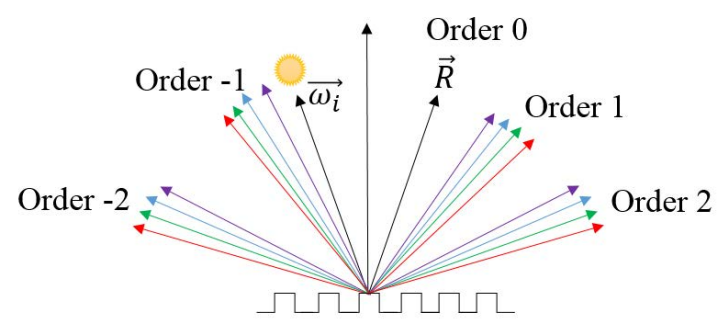

Fig. 3. Orders of diffraction due to reflection off a grating. Light is diffracted according to the visible spectrum from violet to red. Orders may overlap to create superposition of colours

complexity of simulating such effects from the perspective of physically based rendering. Diffraction was first studied by Stam [1999], who introduced a diffraction shader for rendering applications. Building upon Stam's work, a few physically based reflectance models and rendering techniques have subsequently been developed for diffraction effects. However, these suffer from either being computationally expensive due to required wave/Fourier optics simulations, or require extensive pre-computation for real-time rendering [Dhillon et al. 2014]. Secondly, these reflectance models and rendering approaches typically require a height field of the diffraction grating as an input. Since these gratings consist of microscopic structures not visible to the naked eye, they cannot be easily measured with typical reflectance capture setups. In fact, most previous work in computer graphics has focused on statistical or analytic modeling of common grating distributions for this reason. Dhillon et al. [2014] were recently the first to do measurements of diffraction gratings for a realistic rendering application. However, they employed a very specialized Atomic Force Microscope (AFM) for accurate measurement of diffractive microstructures for a biological application. We note that materials that exhibit diffraction effects are now becoming increasing common, particularly due to advances in material manufacturing processes. Hence, practical methods for realistic rendering of such effects are becoming increasingly important for various applications.
In this work, we aim to overcome some of the above challenges and propose both a practical measurement approach as well as an efficient measurement-based rendering technique for realistic rendering of complex diffraction effects in surface reflectance of planar homogeneous diffractive samples. Our proposed measurement approach only requires a regular digital camera and LED flash illumination, while our proposed data-driven rendering technique only requires a single pre-computed diffraction texture look-up for real-time rendering of complex diffraction patterns. Furthermore, we propose a practical method for observing microscopic diffraction gratings in sharp specular surfaces using out-of-focus "bokeh" photography of specular highlights. We demonstrate how such a bokeh measurement can be interpreted as a grating height field for efficiently driving a standard diffraction shader in conjunction with a first order diffraction approximation. Finally, we also drive analytic solutions for a few special cases of diffraction from our measurements and demonstrate efficient rendering with complex light sources and environments. Overall, our approach provides significant practical benefits for real-time rendering of commonly observed (yet complex) diffraction effects in surface reflectance.

\section{RELATED WORK}

In the following, we discuss the most relevent previous work on diffraction and iridescence effects in computer graphics.

\subsection{Diffraction effects in reflectance}

Stam [1999] introduced a physically based reflectance model for diffraction derived from Kirchhoff theory. Stam's model is an extension of the He-Torrance model [He et al. 1991] to include anistropy and requires as an input a height field $h$ that describes the microscopic variations of height of the diffraction grating. The diffraction contribution in the BRDF comes from a $p$ function that is dependent on both the height-field $h$ and the viewing and incoming light directions and has to be calculated at every frame of an animation. As a result, the general form of the BRDF cannot be computed at real-time framerates since it requires the computation of a two-dimensional Fourier transform of the correlation of the $p$ function. 
Sun et al. [2000] rendered iridescence in optical discs by modelling the grooves on a CD as consecutive spheres. Although their method produces realistic results of iridescence on optical disks, the approach is specific to CDs and cannot be extended to other types of diffraction gratings.

Agu and Hill [2002] derived an analytic diffraction model for reflectance based on the Huygens-Fresnel principle. Their BRDF focuses on multislit diffraction along one dimension, i.e, diffraction produced by a periodic microstructure made of rectangles. Although this BRDF is simpler than those based on Fourier optics, the model is limited as it cannot be used to render arbitrary diffraction patterns. We employ a variant of this model in our work to render diffraction in some special cases and estimate a few model parameters from our measurements.

Lindsay and Agu [2006] have proposed adaptively sampling the diffractive BRDF into spherical harmonics $(\mathrm{SH})$ basis for real-time rendering. Their method better preserves the peaks of the diffraction and the dynamic range for rendering and allows integration over the entire hemisphere of illumination. However, the reconstructed diffraction pattern does not preserve high frequencies due to usage of low order $\mathrm{SH}$ basis for lighting and BRDF.

More recently, Cuypers et al. [2012] have proposed a very general reflectance model for diffraction based on formulation of a wave BSDF using Wigner distributions that supports both direct and multi bounce simulations of diffraction using a ray-tracing framework Similar to Stam's model, this approach requires the underlying microstructure of the grating as input in order to carry out the simulation of various forms of diffraction and interference.

Dhillon et al. [2014] recently proposed a reformulation of Stam's BRDF for real-time rendering. Their method uses a Taylor expansion to break the dependency between the height field and the viewing and incoming light direction. This allows a precomputation of diffraction look up tables (terms of the Taylor expansion) using windowed Fourier transforms that can be employed for real-time rendering. Compared to Dhillon's approach which requires many (30-80) lookup tables, our data-driven rendering approach requires pre-computation of just a single diffraction look-up table based on a first order (Born) approximation. Dhillon et al. also propose a measurement approach to record diffraction microstructures of biological specimens (snake skin) using a specialized Atomic Force Microscope (AFM). Although very accurate, such a measurement approach requires very specialized laboratory equipment that is not commonly available. The AFM also relies on physical contact with the diffractive sample in order to make very precise measurements of the surface microstructure. Some highly specular diffractive materials such as LCDs or CDs have a transparent (glass or plastic) layer covering the diffractive (grating) layer making them unsuitable for measurement with such an approach. Instead, we propose a practical method to measure the diffraction grating of such specular samples just using commodity digital photography equipment.

Kang et al. [2015] have recently proposed an RGB diffraction model that avoids full spectrum computations and evaluates a microfacet BRDF model at different rotations of the half-vector for each color channel, producing a separation of colours. Although very simple to implement, their model does not guarantee physically correct rendering. This is because depending on the rotation of half-vectors, the diffracted colours may not happen in the right order. Besides, their method only applies for modeling anisotropic materials as a rotation of the half-vector does not have any effect for an isotropic material.

Also related is the recent work of Dong et al. [2015] who propose employing a profilometer to measure the microstructure of metallic surfaces and drive both microfacet BRDF as well as Kirchhoff scattering (wave optics) based BRDF model for rendering the aggregate surface appearance. Although the microgeometry measurements with a profilometer are down to the scale of the wavelength of visible light, diffraction effects were not modelled in this work.

\subsection{Other wave effects}

Researchers have investigated other iridescent and wave effects more broadly in computer graphics. Sun et al. [2000; 1999a; 1999b] have extensively studied full spectral rendering for modeling iridescence due to thin film interference. In subsequent work, Sun [2006] proposed an RGB-based renderer for efficient simulation of biological iridescence. Granier and Heidrich [2003] have also proposed a simplified RGB-based BRDF model for modeling iridescence in layered materials. Imura et al. [2009] have proposed rendering structural colors in reflectance due to thin film or multi-layer interference as well as diffraction in a unified framework of optical path differences in microstructure. Also related is the work of Hullin et al. [2010] who employed spectral BRDF measurements for modeling bispectral materials exhibiting fluorescence.

Besides iridescence in material reflectance, researchers have studied other iridescence effects in nature. Sadeghi et al. [2012] have proposed a physically-based model for simulation of rainbows that goes further than standard Lorenz-Mie theory (spherical particle assumption) [Frisvad et al. 2007] to also model wave effects due to non-spherical water droplets in the atmosphere. Hullin et al. [2011] have proposed a rendering method and pipeline to simulate physically based lens flare including dispersion and diffraction effects in lenses in real-time. All these methods explicitly simulate the wave optics effects whereas ours employs a direct measurement of surface diffraction to avoid expensive calculations at runtime.

Researchers have also studied wave effects more broadly in the context of audio and visual simulations. Tsingos et al. [2001] have considered diffraction effects due to presence of occluders for sound propagation and simulation using a ray tracing approach. Ziegler et al. [2008] have proposed wave-based occlusion handling for enhancing the realism of digital holography and efficient real time relighting of holograms. They employ the scalar theory of diffraction to simulate defocusing and diffraction caused by an occluder. However their method is specific to computer generated holograms. Oh et al. [2010] have proposed an augmented light field framework to model transmissive diffraction effects due to an occluder using a ray based formulation which does not require simulation of wavefront propagation. Similarly, Wu and Zheng [2013] have proposed a ray-based approach to simulate wave effects in participating media exhibiting absorption and scattering. In contrast, Musbach et al. [2013] have proposed full wave modeling of light propagation and reflection using finite difference time domain method to simulate the advancement of electric and magnetic fields in a 3D scene. Wave effects have also been considered by Levin et al. [2013] for fabricating 
BRDFs with high spatial resolution, and by Ye et al. [2014] for creating custom BxDF displays based on multilayer diffraction. These works aim at fabricating structures that match given reflectance characteristics whereas in this work we measure microstructures to reproduce diffraction effects in surface reflectance.

\section{OVERVIEW}

Diffraction in surface reflectance is a complex optical phenomenon that presents two primary challenges for rendering: measurement and computation. In this work, we tackle these challenges by proposing practical measurement setups and efficient measurement based rendering techniques for complex diffraction effects. Our two primary contributions in this work are as follows.

- We propose a practical approach for direct measurement of diffraction effects in surface reflectance of planar homogeneous samples and a novel rendering technique to efficiently render the measured diffractive irradiance in real-time using a single texture look-up. The approach re lies on the Huygens-Fresnel principle in conjunction with a first order approximation and can enable full spectral rendering and simulate complex light sources using efficient precomputation.

- We propose a novel optical setup to practically observe the diffraction grating of highly specular planar samples using a DSLR camera equipped with a standard zoom lens. We demonstrate how bokeh photography of a specular highlight with this setup can be employed as a height field to efficiently drive a diffraction shader with a first order approximation.

Besides the above primary contributions, we also demonstrate how our measurements can be employed for rendering some special cases with an analytic multislit diffraction solution [Agu and Hill Jr 2002]. The rest of the paper is organised as follows: in Section 4 , we present our novel data-driven rendering approach for rendering complex diffraction patterns using a single pre-computed look-up table. Not all diffractive samples however allow direct measurement of the diffraction pattern due to limited sample size or due to large spatial spread of the pattern. Moreover, regular diffraction shaders instead require the underlying diffraction grating height field as input for Fourier optics simulation of diffraction. Hence, in Section 5 we present how the diffraction grating of highly specular planar samples can be practically observed using bokeh photography and how the data can be interpreted as a height field for diffraction shaders. Finally, in Section 6, we present additional rendering results including rendering with complex light sources and rendering with analytic solutions for a few special cases.

\section{DATA-DRIVEN RENDERING OF DIFFRACTION}

We propose a novel data-driven approach to efficiently render complex diffraction effects in real-time using a single texture look-up based on the measured irradiance. Our rendering technique exploits the Huygens-Fresnel principle in conjunction with a first order Taylor approximation to arrive at our proposed data-driven diffraction
BRDF model. In the following, we first briefly present the mathematical formulation before describing our rendering model and measurement approach.

\subsection{Huygens-Fresnel principle and irradiance}

We first derive an expression for the irradiance of a diffraction pattern based on the well known Huygens-Fresnel principle. The principle states that any point on a wavefront acts as a secondary source and emits a new spherical wave with same frequency, amplitude and phase as the original wave and these new spherical waves all sum up in order to create the wavefront at a later time [Cowley 1995; Ersoy 2006]. Hence in the case of reflection on a diffractive surface, each point $P(x, y)$ creates a spherical wavelet whose contribution has to be summed up over the entire surface.

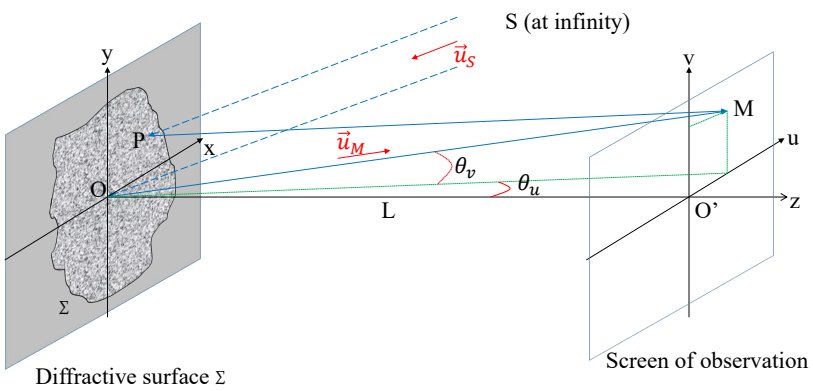

Fig. 4. Diffraction produced by reflection on a diffractive surface $\Sigma$ (with height field $h$ ) and observed on a screen at a distance $L$.

Mathematically, given reflection on a diffractive surface $\Sigma$ with height field $h$, the complex amplitude observed at a point $M(u, v)$ on a screen at a distance $L$ from the surface (see Fig. 4 ) is given by:

$$
a(M, \lambda)=\frac{i s_{o}}{\lambda L} e^{i \omega t} \iint_{\Sigma} h(x, y) e^{i k \delta_{S P M}} d x d y,
$$

where $k=\frac{2 \pi}{\lambda}$ is the spatial frequency of the wave with wavelength $\lambda$ and $s_{o}$ is the amplitude of the light source $S$ and $\delta_{S P M}$ is the optical path between the points $S, P$ and $M$. We assume Fraunhofer (far-field) diffraction to apply in this setting with the screen and the light source $S$ far from the diffractive surface $\Sigma$.

Equation 1 can be expanded as follows based on a first order approximation of the optical path $\delta_{S P M}$ (see Appendix A):

$a(M, \lambda)=\frac{i s_{o}}{\lambda L} e^{i\left(\omega t+k\left(\delta_{S O}+L\right)\right)} \iint_{\Sigma} h(x, y) e^{-i k\left(\frac{x u+v y}{L}-\overrightarrow{O P} \cdot \vec{u}_{S}\right)} d x d y$

A change in parametrization using the angles $\theta_{u}$ and $\theta_{v}$ (Eq. 3) and $\overrightarrow{O P} \cdot \vec{u}_{S}=-(x \xi+y \eta)$ with $\vec{\omega}_{i}=-\vec{u}_{S}=(\xi, \eta, \epsilon)$ lead to the final expression of the amplitude (Eq. 4) where $H$ is the Fourier transform of $h$. This is valid using small angles approximation for reflection close to normal incidence.

$$
\begin{gathered}
\sin \left(\theta_{u}\right) \approx \tan \left(\theta_{u}\right)=\frac{u}{L} \text { and } \sin \left(\theta_{v}\right) \approx \tan \left(\theta_{v}\right)=\frac{v}{L} \\
a(M, \lambda)=\frac{i s_{o}}{\lambda L} e^{i\left(\omega t+k\left(\delta_{S O}+L\right)\right)} H\left(k\left(\xi+\sin \theta_{u}\right), k\left(\eta+\sin \theta_{v}\right)\right)
\end{gathered}
$$


We can finally derive an expression for the diffraction irradiance $I(M, \lambda)$ that is measured on the screen as the square module of the complex amplitude $I(M, \lambda)=<a(M, \lambda) a^{*}(M, \lambda)>$ :

$$
I(M, \lambda)=\left(\frac{s_{o}}{\lambda L}\right)^{2}<\left|H\left(k\left(\xi+\sin \theta_{u}\right), k\left(\eta+\sin \theta_{v}\right)\right)\right|^{2}>
$$

Note that a sensor such as our eyes or a camera directly measures the above diffraction irradiance pattern. The pattern is mostly invariant to the angle of incidence of the light source, with the only effect being that the pattern is translated in the plane $(u, v)$ by $(\xi, \eta)$. Let $\vec{t}$ and $\vec{b}$ denote the tangent and bitangent of the diffractive surface. Then the irradiance can also be parameterized in terms of the vector $\vec{h}=\overrightarrow{u_{M}}-\overrightarrow{u_{S}}$ as follows

$$
I(k(\vec{h} \cdot \vec{t}), k(\vec{h} \cdot \vec{b}))=\left(\frac{s_{o}}{\lambda L}\right)^{2}<|H(k(\vec{h} \cdot \vec{t}), k(\vec{h} \cdot \vec{b}))|^{2}>
$$

Eq. 6 forms the basis of our data-driven rendering approach. For simplicity, the term $\left(\frac{s_{o}}{L}\right)^{2}$ is omitted in the next sections as it only affects the brightness of the final rendering depending on the light source distance $(L)$ and intensity $\left(s_{0}\right)$.

\subsection{Rendering model}

Our rendering approach takes into account the diffuse, specular and diffractive components of the BRDF $f_{r}$ separately (Eq. 7).

$$
f_{r}\left(\vec{\omega}_{i}, \vec{\omega}_{o}\right)=\text { diffuse }+ \text { specular }+ \text { diffraction }
$$

For rendering reflections on regular surfaces, we compute the diffuse and specular components using a standard geometric optics BRDF model for isotropic reflections such as Cook-Torrance [1982]. For rendering diffractive surfaces, we employ a simplified diffraction model based on a first order (Born) approximation of Stam's model [1999] (see Appendix B):

$$
f_{r, \text { diffraction }}\left(\vec{\omega}_{i}, \vec{\omega}_{o}, \lambda\right)=\frac{4 \pi^{2} F^{2} G}{\lambda^{4}}<|H(k u, k v)|^{2}>
$$

Here, $H$ is the Fourier transform of the height map $h$, and $F$ and $G$ are the standard Fresnel and geometric terms in Stam's model. This can be further expressed in terms of the irradiance function (Eq. 6) as:

$$
f_{r, \text { diffraction }}\left(\vec{\omega}_{i}, \vec{\omega}_{o}, \lambda\right)=\frac{4 \pi^{2} F^{2} G}{\lambda^{2}} I(k(\vec{h} \cdot \vec{t}), k(\vec{h} \cdot \vec{b}))
$$

Note that the $I(k(\vec{h} \cdot \vec{t}), k(\vec{h} \cdot \vec{b}))$ term in the above BRDF model takes into account the diffraction for a single wavelength. For rendering, we employ its formulation $S_{d}(\vec{h})$ that integrates the diffracted irradiance for each wavelength in the visible spectrum $\left[\lambda_{\min } ; \lambda_{\max }\right]$ (Eq. 10 and 11), assuming that the Fresnel term does not depend on the wavelength :

$$
\begin{gathered}
f_{r, \text { diffraction }}\left(\vec{\omega}_{i}, \vec{\omega}_{o}\right)=4 \pi^{2} F^{2}\left(\vec{\omega}_{i}, \vec{\omega}_{o}\right) G\left(\vec{\omega}_{i}, \vec{\omega}_{o}\right) S_{d}(\vec{h}) \\
S_{d}(\vec{h})=\int_{\lambda_{\min }}^{\lambda_{\max }} \frac{1}{\lambda^{2}} I\left(\frac{2 \pi}{\lambda} \vec{h} \cdot \vec{t}, \frac{2 \pi}{\lambda} \vec{h} \cdot \vec{b}\right) d \lambda
\end{gathered}
$$

Such a diffraction term can be calculated efficiently in real-time by precomputing $S_{d}$ into a lookup table and accessing it as a texture in a GLSL fragment shader. The calculations are done in the XYZ colour space to accurately take into account the spectrum of the light source in the scene. Given the spectral power distribution of a light source $P$ and the CIE colour matching functions $\bar{x}$, the diffracted irradiance for the $X$ component is given by Eq. 12. Similar computations can be done for the $\mathrm{Y}$ and $\mathrm{Z}$ components.

$$
S_{d, X}(\vec{h})=\int_{\lambda_{\min }}^{\lambda_{\max }} \frac{1}{\lambda^{2}} I\left(\frac{2 \pi}{\lambda} \vec{h} \cdot \vec{t}, \frac{2 \pi}{\lambda} \vec{h} \cdot \vec{b}\right) P(\lambda) \bar{x}(\lambda) d \lambda
$$

We employ this formulation to index the $S_{d}$ lookup table in a fragment shader for our data-driven BRDF model.

\subsection{Imaging and precomputation}

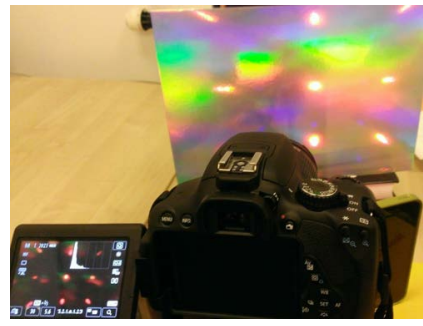

(a)

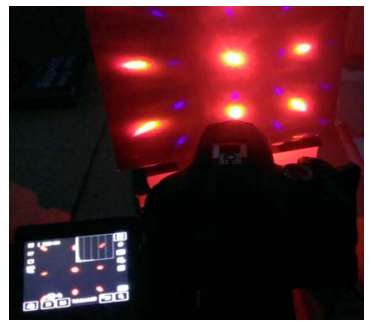

(b)
Fig. 5. (a) Measurement setup shown with the house lights switched on (b) An LED flash illuminates the sample with a red spectral filter for measurement of the diffraction pattern.

We now describe our measurement approach for imaging diffraction effects. We make the observation that for many common planar homogeneous diffractive samples, the diffraction pattern can be directly observed on the sample when it is illuminated with a point light source. We employ a regular DSLR camera (Canon 650D) with a standard lens (EF-S 18-55mm IS II) for our measurements and a smartphone (iPhone 5S) LED flash as the light source in our setup (Fig. 5). We adjust the light source distance from the sample such that the diffraction pattern is entirely visible on the sample. The distance typically varied from 10 centimeters to 3 meters in our measurements. We also attach a narrow band spectral filter (Roscolux thin film sheet) in front of the LED flash in order to restrict the diffraction measurement to nearly a single wavelength. We empirically chose a red filter (peak transmission at $680 \mathrm{~nm}$ ) and in some cases a green filter (peak transmission at $520 \mathrm{~nm}$ ) for our measurements as these provided us with the good color isolation and clean measurements. We also used only a single color channel of the camera to have even better spectral separation. With this setup, we take a high-dynamic range (HDR) photograph [Debevec and Malik 1997] of the diffraction pattern and remove the black levels. Fig. 6 shows two examples of data captured with this setup (also see supplemental material).

Our measurement corresponds to reflected radiance $L_{o}$ due to incident radiance $L_{i}$ from the light source:

$$
L_{o}(\vec{h}, \lambda)=\frac{4 \pi^{2} F^{2} G}{\lambda^{2}} I\left((k(\vec{h} \cdot \vec{t}), k(\vec{h} \cdot \vec{b}))\left(\vec{n} \cdot \vec{\omega}_{i}\right) L_{i}(\lambda)\right.
$$

The measurement is done as close to normal incidence as possible $\left(G \approx 1\right.$ and $\left.\vec{n} \cdot \vec{\omega}_{i} \approx 1\right)$ with unit incident radiance (after radiometric 


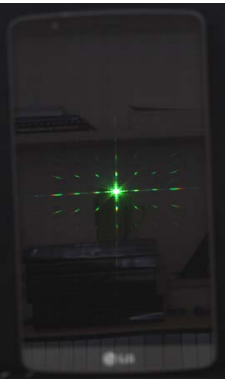

(a)

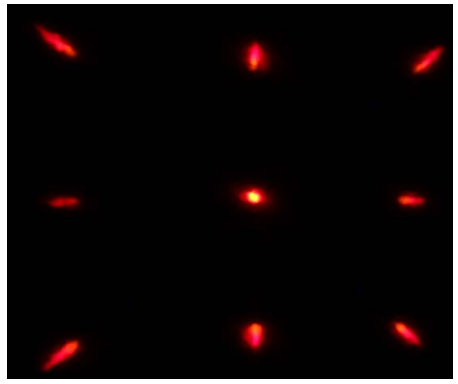

(b)
Fig. 6. Photograph of diffraction patterns. (a) LG phone with a green spectral filter. (b) Holographic paper with a red filter.

calibration), and for a single wavelength $\lambda_{m}$. Therefore we measure the quantity :

$$
L_{o, m}\left(\vec{h}, \lambda_{m}\right)=\frac{4 \pi^{2} F_{0}^{2}}{\lambda_{m}^{2}} I\left(\frac{2 \pi}{\lambda_{m}} \vec{h} \cdot \vec{t}, \frac{2 \pi}{\lambda_{m}} \vec{h} \cdot \vec{b}\right)
$$

where $F_{0}$ is the Fresnel factor at normal incidence.

The next step is to compute the diffraction pattern under any arbitrary illumination spectrum from the photograph captured under narrow band illumination. The photograph provides a measurement of the irradiance for all $(\vec{h} \cdot \vec{t}, \vec{h} \cdot \vec{b})$ and a given wavelength $\lambda_{m}$. First, we detect the center of the specular reflection as the center of the lobe having the maximum brightness in the image. This is the order 0 of diffraction. We then sample the range $[-1 ; 1] \times[-1 ; 1]$ to sample the values of $(\vec{h} \cdot \vec{t}, \vec{h} \cdot \vec{b})$. For each sampled value, we calculate the integral of Eq. 15 using the trapezoidal rule. As the reflected radiance $L_{o, m}$ has been measured for a given wavelength $\lambda_{m}$, we evaluate it at $L_{o, m}\left(\frac{\lambda_{m}}{\lambda} \vec{h}, \lambda_{m}\right)$ in the integral. This way, we estimate the intensity function for any wavelength from a single measurement at one unique wavelength and employ this to compute the $S_{d}$ lookup table.

$$
S_{d, X}(\vec{h})=\frac{1}{4 \pi^{2} F_{0}^{2}} \int_{\lambda_{\min }}^{\lambda_{\max }} \frac{\lambda_{m}^{2}}{\lambda^{2}} L_{o, m}\left(\frac{\lambda_{m}}{\lambda} \vec{h}, \lambda_{m}\right) P(\lambda) \bar{x}(\lambda) d \lambda
$$

The final output of the above steps is a lookup table $S_{d}$ sampled in $[-1 ; 1] \times[-1 ; 1]$. Similarly to [Dhillon et al. 2014], a non linear sampling may be required when the diffraction happens very close to the specular reflection (e.g., in LCD screens). Such a sampling is given by $\left(u_{l}^{n}, v_{l}^{n}\right)$ with $\left(u_{l}, v_{l}\right)$ sampled uniformly in $[-1 ; 1] \times[-1 ; 1]$ and $n$ an odd power (typically 5 ). The above computation of the lookup table is very efficient and computing a $2048 \times 2048$ lookup table using an HDR photograph of the diffraction pattern of size $1000 \times 1000$ took only a few seconds on an Intel i7-4720HQ $(2.6 \mathrm{GHz})$ laptop.

\subsection{Rendering results}

We now present some diffraction patterns rendered with our datadriven rendering approach. The renderings were computed assuming a full white illumination spectrum but can be calculated for any spectral distribution. Additional examples are presented in Section 6.

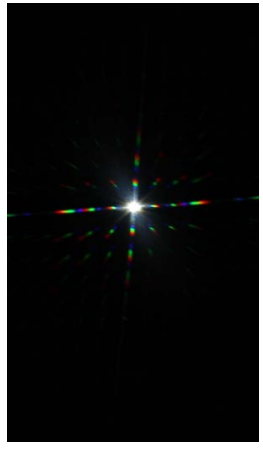

(a)

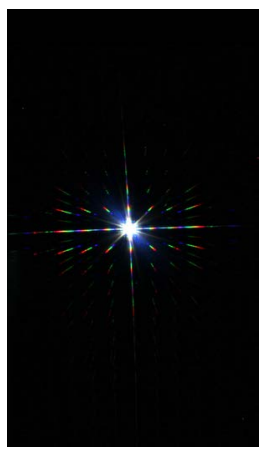

(c)

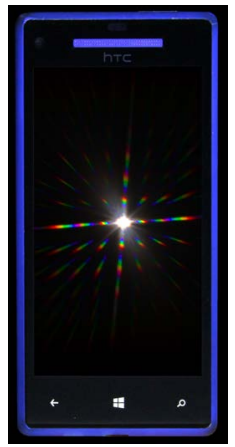

(b)

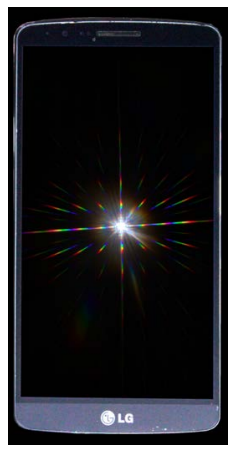

(d)
Fig. 7. Rendered diffraction patterns of phones. Top-row: HTC 8X. Bottomrow: LG G3. (a, c) Photograph. (b, d) Rendering.

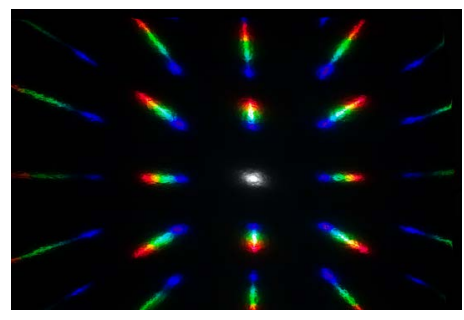

(a)

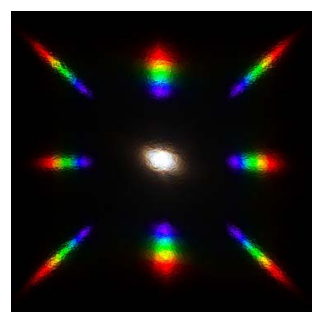

(b)
Fig. 8. Diffraction pattern of a holographic paper. (a) Photograph. (b) Rendering.

Figure 7 presents side by side comparisons between the renderings of diffraction pattern produced by smartphone LCD screens using our method and a photograph. The two smartphones exhibit different diffraction patterns that are accurately rendered. Here, the basic reflectance maps for the two phones were measured using polarized second order gradient illumination emitted by a desktop LCD panel according to [Ghosh et al. 2009]. Here, the phone bodies are rendered using Cook-Torrance BRDF with measured diffuse and specular albedo, while the screens are rendered with our data-driven diffraction model replacing the specular component of the BRDF. 


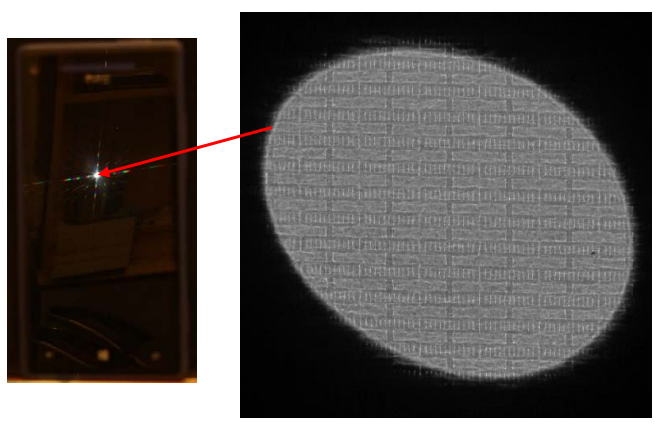

(a)

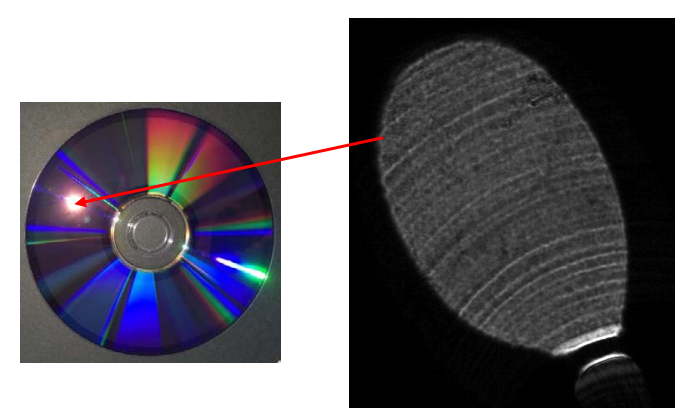

(b)

Fig. 9. (a) Pixels of an HTC smartphone LCD screen seen through a bokeh. (b) Tracks on a CD-R seen through a bokeh. Note that only the blue channel of the bokeh is used as blue wavelength is observed to have the sharpest detail and least scattering.
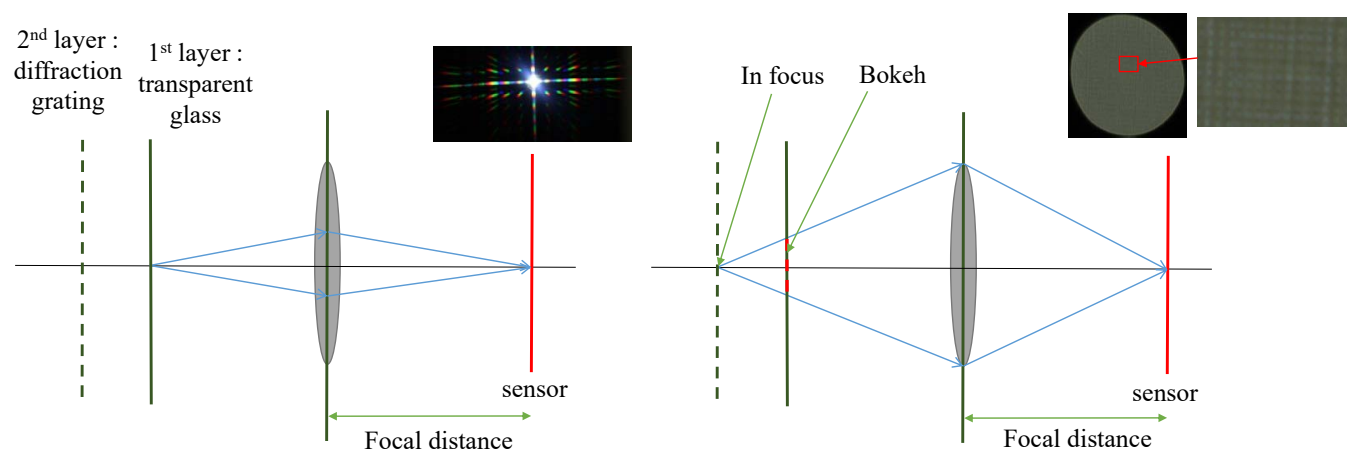

Fig. 10. (Left) The first layer of the sample is in focus (e.g., glass on a smartphone LCD). The specular highlight and diffraction pattern are visible. (Right) The first layer of the sample is out of focus, but the second layer becomes in focus revealing the diffraction grating.

Figure 8 is a side by side comparison between our method and a photograph of the diffraction pattern produced by a holographic paper. Unlike the phone screens, the holographic paper is rough specular making wider lobes of diffraction. Here, we modeled the the bumpiness of the holographic paper's surface from measurement using step-edge illumination according to Wang et al. [2011]. Note that the rendering only exhibits up to $1^{\text {st }}$ order diffraction compared to the photograph where some $2^{\text {nd }}$ order diffraction is also visible. This is because the rendering is driven by a measurement which only included up to $1^{\text {st }}$ order diffraction in the pattern visible on the sample with the specific spectral filter (Fig. 6 b). We present alternate renderings of the pattern which include $2^{\text {nd }}$ order diffraction in Section 6 . Note that in the above renderings, the central specular highlight ( $0^{\text {th }}$ order diffraction) is also included in the measured diffraction pattern and hence rendered with the proposed datadriven approach.

\section{BOKEH PHOTOGRAPHY OF DIFFRACTION GRATINGS}

In the previous section, we presented a novel data-driven rendering approach that relies on direct observation of the diffraction irradiance pattern on the surface of a planar homogeneous sample. However, such direct observation of the irradiance pattern is not always possible on a sample, e.g., due to limited sample size or due to large spatial spread of the pattern relative to the sample. Moreover, typical diffraction shaders instead require the underlying grating structure as input for Fourier optics simulation of the resulting diffraction. The size of microstructures in typical diffraction gratings are around the micrometer. Hence, their accurate measurement usually requires highly specialized microscopy equipment. For instance, Dhillon et al. [2014] have employed an AFM which has the advantage of not being diffraction limited at such microscopic scales compared to an optical setup. However, besides being a very specialized and expensive equipment, an AFM relies on direct physical contact with a diffractive surface in order to measure its microstructure. Some highly specular diffractive materials such as LCD screens or optical discs have a transparent (glass or plastic) layer covering the diffractive layer making them unsuitable for direct contact based measurement. Instead, for such common highly specular diffractive surfaces we propose a practical method to measure the underlying diffraction grating by photographing the bokeh of a specular highlight seen on the sample surface. 


\subsection{Measurement}

Our measurement approach simply employs a regular DSLR camera equipped with a standard zoom lens for high resolution imaging of the bokeh and a point light source for illuminating the sample surface. In this case, we equip the camera (Canon 650D) with an entry level 55-250 mm zoom lens (Canon EF-S f/4-5.6 IS STM) which allows focus at a relatively short distance of 0.85 meters ${ }^{1}$. We place the camera around 85 centimeters from the sample and illuminate the shiny diffractive sample with a phone (iPhone 5S) LED flash from a distance of around 3 meters. Additionally, we set the camera to the largest aperture setting allowed by the lens in this setup (f/5.6) as we desire a shallow depth of field. We then zoom as much as possible on to the specular highlight on the sample due to the LED flash and go out-of-focus on the specular highlight to capture its bokeh. What appears on the camera sensor in this setting is a high resolution image of the underlying diffraction grating. It should be noted that the grating is rotated by $90^{\circ}$ on the sensor due to the rotation of the lens focus ring for out-of-focus photography. We invert this rotation in post-process. Two examples of diffraction gratings seen through a bokeh are shown in Fig. 9 (also see supplemental material).

Such an imaging mechanism has similarity with confocal imaging [Wilson et al. 1998]. In our case, when the camera is in focus on the first (transparent) layer, both the specular highlight and the diffraction pattern are visible. However, when the camera goes outof focus on the specular highlight, the first layer becomes blurry but the second layer (the diffraction grating) becomes visible (Fig. 10). Note that Mohan et al. [2009] have proposed a related method that uses a bokeh to observe tiny barcodes called bokodes. However, their method uses backlighting for the barcodes and a specific magnification lens is mounted in front of the bokodes to make them visible to a camera.

\subsection{Height field for rendering}

We infer the recorded bokeh images as the height field of the underlying diffraction grating for rendering with a diffraction shader Here, we simply infer the recorded image intensity (up to an affine transformation) as surface height field based on a standard "darkis-deep" heuristic typically employed in shape-from-shading. This is based on the observation that the measured diffractive surface patch inside the bokeh can be assumed to be uniformly lit at this microscopic scale by the spatial PSF of the out-of-focus specular highlight. In practice we interpret only the observation of the blue channel (shortest wavelength) of the bokeh, which we observe to contain the sharpest detail ${ }^{2}$, as the height field.

The above can be seen as a geometric optics interpretation of the proposed bokeh measurement. Interestingly, there is also a Fourier optics interpretation of the above measurement which can once again be motivated by the Born (first order) approximation of a diffraction BRDF. In the case of the Born approximation, the spectral density of the underlying height field can be directly "seen" in

\footnotetext{
${ }^{1}$ A similar entry level zoom lens with a larger zoom factor such as the Canon EF Telephoto 75-300 mm - f/4.0-5.6 III USM does not work as well since its minimum focal distance is 1.5 meters.

${ }^{2}$ Any optical system is diffraction limited and therefore cannot resolve two points that are very close according to the Rayleigh criterion. The smaller the wavelength, the higher the resolution that can be imaged [Cowley 1995].
}

the specular highlight [Stam 1999]. This implies that the visible specular lobe can be approximated as the result of an optical Fourier transform (FT) of the underlying height field $h$. Furthermore, in the case of measurement (a real valued function), the inverse Fourier transform (IFT) is just the conjugate of the FT with equal magnitude and opposite phase. According to Fourier optics theory of lenses [Stark 2012], if a transmissive object is in the focal plane of a lens, then the lens computes the Fourier transform of the transmission function in its other focal plane. The process of bokeh photography can be seen as imaging the other focal plane of the lens optically recording the FT of the transmission function. Thus, with the Born approximation, our proposed bokeh photography of the specular highlight can be seen to compute the IFT of the spectral density function with an optical FT (only magnitude not phase), revealing the grating height field $h$.

Based on the above insights, the bokeh measurements can be employed as grating microstructure in conventional diffraction shaders. In practice, we need to first apply deconvolution to the measured grating structure because the lens aperture acts as a low pass filter on the measured signal [Stark 2012] ${ }^{3}$. We employ Richardson-Lucy deconvolution (Matlab) on the bokeh images (assuming a Gaussian PSF with empirically chosen parameters, see Fig.11) and an affine transformation obtained from measurements of a known grating (see Appendix C) to obtain the height fields for renderings. We need one additional calibration step in order to drive a diffraction shader with our measurement which is the spatial resolution at which the grating is sampled with our imaging setup. We infer this unknown spatial scale and affine transformation for interpreting measured intensities as height field from similar bokeh measurement of a known reflective diffraction grating. This step is explained in more detail in Appendix C.

Fig. 12 shows diffraction patterns generated for two types of smartphone LCD screens (HTC 8X and LG G3) by employing the deconvolved bokeh measurement as a height field according to the rendering method of Dhillon et al. [2014]. However, instead of a higher order Taylor approximation employed by Dhillon et al. for rendering natural biological structures, we simply employ a first order Taylor approximation to render the diffraction pattern of the periodic gratings that we image with the bokeh technique. This requires us to only store three pre-computed look-up tables $\left(1^{\text {st }}\right.$ order Taylor terms) for real time rendering of the diffraction pattern with the approach of [Dhillon et al. 2014].

Fig. 13 shows comparison renderings of diffractions patterns generated for the LG G3 grating with increasing orders of Taylor approximation. As can be seen for the periodic structure of the phone LCD screen, the first order Taylor approximation provides a good qualitative result and in fact higher order Taylor terms make the rendering result worse due to additional high frequencies that appear when the Taylor expansion has not yet converged (we refer to [Dhillon and Ghosh 2016] for a more detailed disussion of this phenomenon). This implies that diffraction patterns of period grating structures can be efficiently rendered in a diffraction shader using a low order approximation. In fact, Chebyshev polynomials [Dhillon

\footnotetext{
${ }^{3}$ The larger the lens aperture diameter, the smaller is the effect of the blur due to the lens pupil. We employ the largest aperture setting (f/5.6) allowed on the lens.
} 


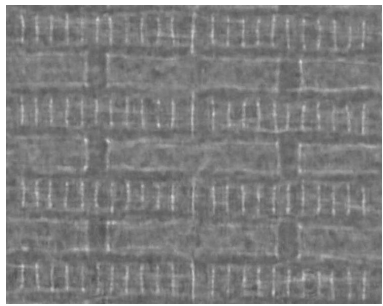

(a)

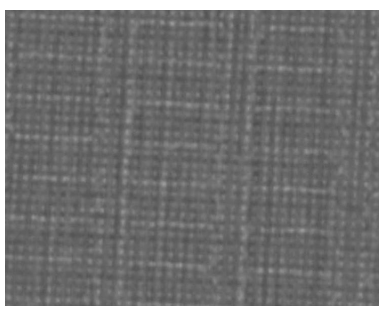

(c)

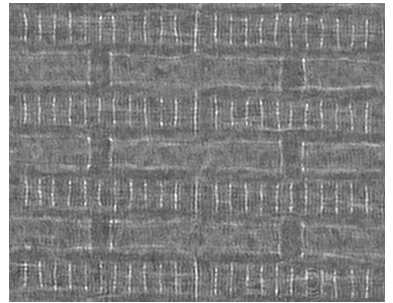

(b)

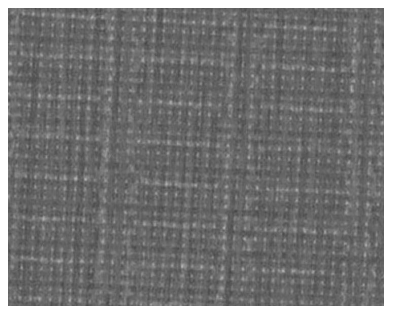

(d)
Fig. 11. Measured bokehs (a, c) vs deconvolved gratings (b, d) used as height field in a diffraction shader. (Top-row) HTC 8X phone. (Bottom-row) LG G3 phone.

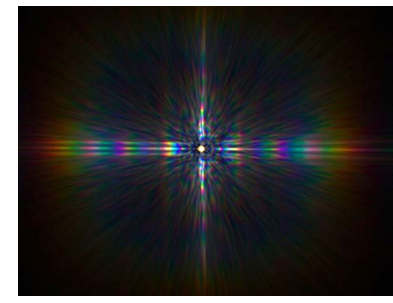

(a)

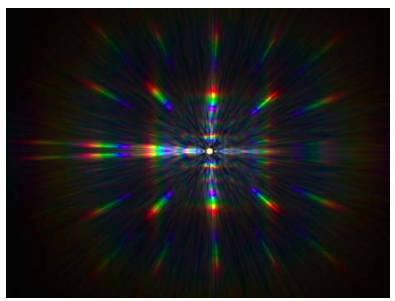

(b)
Fig. 12. Rendered diffraction patterns according to [Dhillon et al. 2014] using the data captured with the bokeh measurement as the grating. (a) HTC 8X. (b) LG G3.

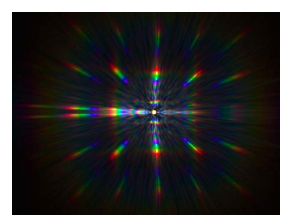

(a)

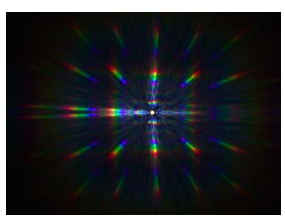

(b)

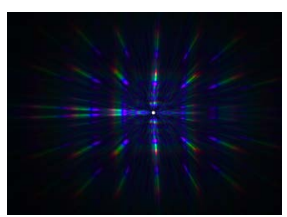

(c)
Fig. 13. Rendered diffraction pattern for the LG G3 grating according to [Dhillon et al. 2014] using increasing orders of Taylor approximation. (a) $1^{\text {st }}$ order. (b) $2^{\text {nd }}$ order. (c) $5^{\text {th }}$ order.

and Ghosh 2016] have been recently employed to represent diffraction more efficiently compared to a Taylor series expansion with only two pre-computed textures shown to sufficiently accurately reproduce complex patterns.

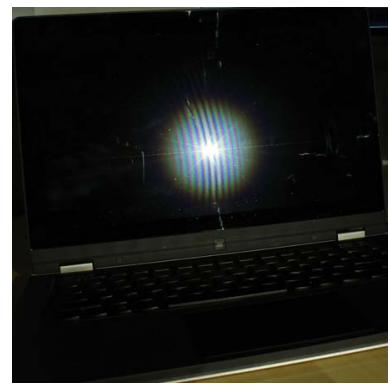

(a)

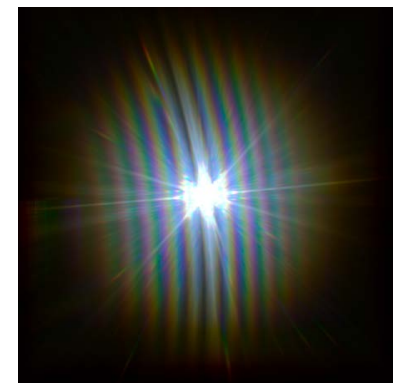

(b)
Fig. 14. Bragg diffraction pattern on a laptop screen. (a) Photograph. (b) Rendering.

Thus, bokeh photography can be used in this manner to practically observe diffraction gratings and to qualitatively produce diffraction effects (Fig. 12). However, we note that sharp high frequencies in the diffraction patterns are not reproduced accurately (compared to photographs in Fig. 7). This is due to the inherent optical limitations (diffraction limited imaging [Levoy et al. 2006]) of the employed commodity photography equipment (see Figs. 28 and 29) for imaging microscopic structures. The diffraction limit of a lens is related to its f-number [Edmund Optics 2016]. With an f/5.6 aperture, our employed lens can theoretically resolve 343 line pairs per millimetre $(\mathrm{lp} / \mathrm{mm})$ at $0 \%$ contrast. A high-end zoom lens such as the Canon EF $70-200 \mathrm{~mm} \mathrm{f} / 2.8 \mathrm{~L}$ IS II USM has an aperture of $\mathrm{f} / 2.8$ and therefore a theoretical diffraction limit of $687 \mathrm{lp} / \mathrm{mm}$. This corresponds to an upper bound of the spatial resolution at which gratings can be imaged with the bokeh method. Hence, we believe the rendering results can be further improved by employing a higher quality zoom lens with a larger magnification factor for imaging grating structures of highly specular diffractive surfaces.

\section{ADDITIONAL RESULTS}

We now present some additional results of acquisition and rendering of diffraction effects with our proposed approaches. All renderings were carried out in real time in a GLSL fragment shader. We achieved 65 FPS running on an Intel-i7 laptop with an entry level NVIDIA GTX 960M graphics card.

First, we present some additional results produced with our proposed data-driven technique (Section 4). Fig. 14 presents rendering of a diffraction pattern observed on a Lenovo Yoga laptop screen compared to the photograph of the pattern seen on the screen. It is interesting to note that the pattern seen here is an example of Bragg diffraction [Cowley 1995], which is the result of diffraction in a crystal lattice causing an interference pattern. A physically based simulation of Bragg diffraction would generally be more computationally expensive given that this is the result of diffraction in a layered structure. Instead, our data-driven rendering approach can efficiently render this pattern without any effect on the rendering framerate. Since we make a narrow band measurement of the diffraction pattern to compute the $S_{d}$ lookup table, our spectral rendering technique can simulate rendering under any arbitrary light 


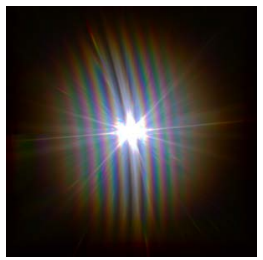

(a)

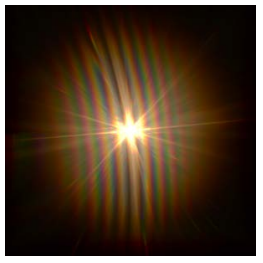

(b)

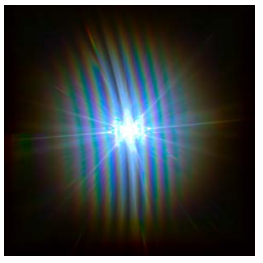

(c)
Fig. 15. Laptop diffraction pattern rendered with different illumination spectra. (a) Full spectrum. (b) Incandescent (CIE Illuminant A). (c) Cool blue (approximated as inverse incandescent).

source spectrum. In Fig. 14 (b), the pattern has been rendered under a fluorescent white spectrum (CIE Illuminant F1) to approximate the spectral power distribution of the LED flash in the photograph (a). Fig. 15 presents rendering of the diffraction pattern under some other common light source spectra.

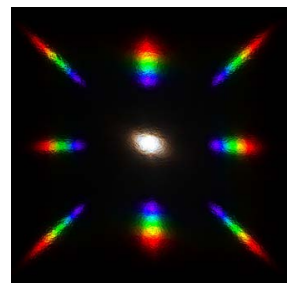

(a)

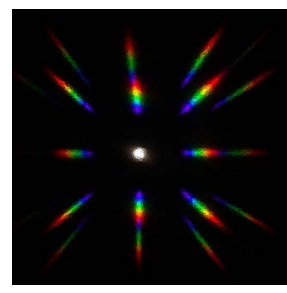

(c)

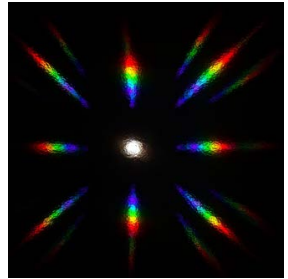

(b)

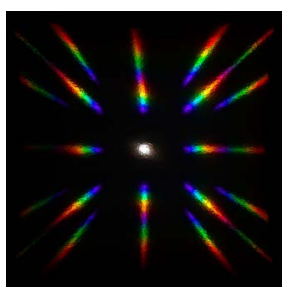

(d)
Fig. 16. Rendering of diffraction pattern on holographic paper with spectral vs RGB measurements. (a) Red spectral filter. (b) Red channel. (c) Green channel. (d) Blue channel.

In Fig. 16, we compare the rendering results of our data-driven approach when using a spectral filter for measuring a narrow band diffraction pattern for the irradiance function $I$, versus using regular broad spectrum LED flash illumination and using the camera's RGB channels for spectral separation. In this holographic paper example, the colors are fairly spread out in the diffraction pattern and simply employing the camera's RGB color channels to approximate spectral measurments works reasonably well in this case. Note that different spectral bands spread out by different distances on the sample during measurement. Hence, renderings employing red spectrum measurements for the irradiance function, either using the red spectral filter (a) or when using the camera red channel (b), only include

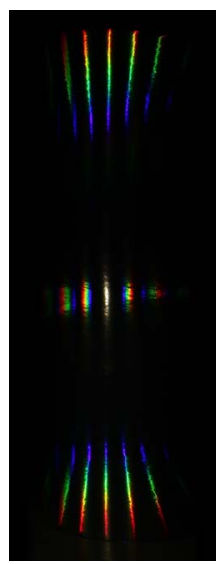

(a)

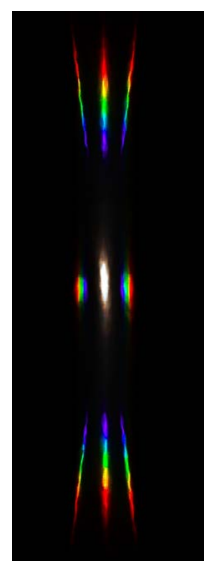

(b)
Fig. 17. Diffraction on the holographic paper bent in a cylindrical shape. (a) Photograph. (b) Rendering. Note that the $2^{\text {nd }}$ order diffraction lobes visible in the photograph are not present in the rendering as they were not recorded in the spectral measurement (see section 4.4).

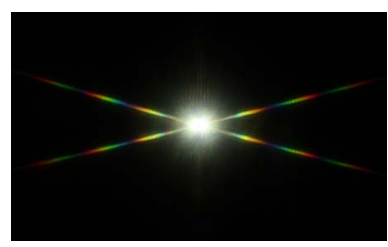

(a)

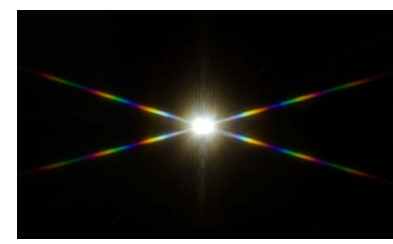

(b)
Fig. 18. Diffraction pattern of a LED TV. (a) Photograph. (b) Rendering.

$1^{\text {st }}$ order diffraction in the rendered pattern. However, renderings employing the green or the blue color channel measurements include some $2^{\text {nd }}$ order diffraction (c, d). As expected, the narrow band spectral measurement results in the most accurate rendering of the pattern (a) when compared to a photograph (Fig. 8, a). Amongst results with camera RGB measurements, the green channel result (c) best matches the photograph in this case.

The measurement method presented in Section 4.3 assumes planar surfaces to record the diffraction pattern. However, the generated lookup table can be used to render any 3D surface as long as the diffraction is homogeneous over the entire surface. Fig. 17 shows such an example where the holographic paper is rolled into a cylinder about its $\mathrm{Y}$ axis resulting in the diffraction pattern (under a point light) getting compressed horizontally. The cylindrical bending of the paper alters the shape of the diffraction pattern which is correctly predicted using our data-driven rendering method.

Fig. 18 presents an additional example of diffraction observed on a modern LED TV (LG 42" smart TV) screen (a) that is rendered with our proposed data-driven rendering approach (b). Note that for the LED TV and other data-driven results presented in the paper, we also render the central specular highlight ( $0^{t h}$ order diffraction) using the measured data. This helps to reproduce the realistic glare around such highlights in rendering. 


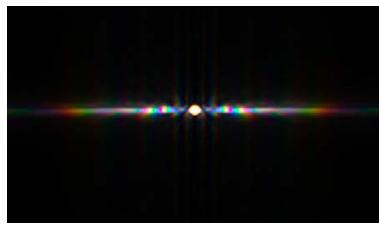

(a)

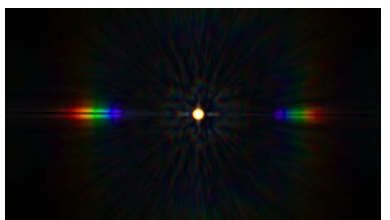

(b)

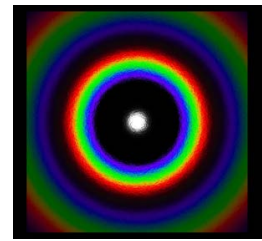

(a)

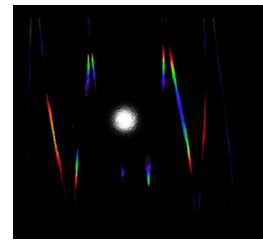

(b)

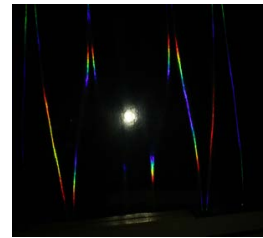

(c)
Fig. 19. Diffraction patterns rendered from grating measurements using bokeh photography. (a) CD. (b) Blazed diffraction grating (ThorLabs).

Finally, Fig. 19 presents additional examples of renderings of diffraction patterns of two highly specular diffractive samples generated from the measured underlying grating structure using the bokeh photography approach described in Section 5 . Here, the diffraction pattern for the $\mathrm{CD}$ (a) was generated using data extracted from a narrow 1D slice (across the tracks) of the bokeh measurement shown in Fig. 9, (b) as the grating. Similarly, the rendering of the blazed grating (b) has been generated using the bokeh measurement shown in Fig. 27 as the underlying grating. Note the asymmetry in brightness of the rendered diffraction lobes about the central specular highlight which is the result of the underlying asymmetric (blazed) height field. We present an alternate way to exploit such bokeh measurement for driving parameters of an analytic solution for diffraction in the next section.

\subsection{Analytic Multislit Rendering}

Thus far, we have presented results of diffraction patterns rendered with our proposed data-driven rendering method, or with our proposed bokeh photography method for measurement of diffraction gratings in the case of highly specular surfaces. However, in some cases, the diffraction pattern on a sample cannot be directly photographed for data-driven rendering, e.g., due to the large spatial spread of the pattern across the sample boundary or due to discontinuities of the pattern on the sample due to spatial variation. Employing the bokeh technique instead to measure the diffraction grating is also not always possible, e.g., if the diffractive region is not sharply specular enough or large enough to image the bokeh. Instead, we note that if the structure of the overall diffraction pattern seen on the sample is fairly simple (e.g., radial or 1D), then in some cases it is possible to apply the well-known analytic function due to multislit diffraction (special case corresponding to diffraction produced by evenly spaced rectangles called slits [Agu and Hill Jr 2002]) to render such patterns.

The analytic solution for one dimensional multislit diffraction has three parameters: the number $N_{x}$ of slits illuminated, the width $w_{x}$ of a slit and the spacing between the slits $\Delta_{x}$. Its expression is given in Equation 16 where sinc denotes the function $\frac{\sin (x)}{x}$.

$$
I\left(\theta, w_{x}, \Delta_{x}, N_{x}\right)=\operatorname{sinc}^{2}\left(\frac{\pi w_{x} \sin (\theta)}{\lambda}\right)\left(\frac{\sin \left(\frac{N_{x} \pi \Delta_{x} \sin (\theta)}{\lambda}\right)}{N_{x} \sin \left(\frac{\pi \Delta_{x} \sin (\theta)}{\lambda}\right)}\right)^{2}
$$

However, unlike Agu and Hill [2002], we employ it to specifically model the diffraction irradiance function $I$ in our first order BRDF model (Equation 9). Fig. 20 presents an example of a holographic
Fig. 20. Example of a radial diffraction pattern limited to narrow vertical stripes on a holographic paper. (a) Diffraction pattern without mask. (b) Rendering with mask. (c) Photograph.

paper that exhibits a radial diffraction pattern that is restricted to a few narrow vertical stripes on the sample (c). We propose to model the radial pattern by rotating a $1 \mathrm{D}$ multislit pattern around its center. We use this rotated function as the irradiance function in Equation 11 to compute the $S_{d}$ lookup table for rendering (a). Additionally, we apply a segmentation mask to restrict the radial pattern to the narrow vertical stripe regions in the rendering (b). We automatically segmented the mask region using a brightness threshold applied to the photograph of the sample under flash illumination (c). Note that in this case, we render the central specular highlight using a Cook-Torrance BRDF lobe. Here, we empirically set the parameters of the $1 \mathrm{D}$ multislit equation to best match the spatial spread of the pattern seen in the photograph of the sample.

Another type of diffractive material that can be well modeled with such an analytic solution is a compact disc (CD). We model the diffraction pattern on a $\mathrm{CD}$ to be caused by one dimensional multislit gratings that are oriented along the tangent $\vec{t}$ orthogonal to the direction of the radial tracks. Hence, the above 1D multislit formula can be used to render diffraction in CDs in the following way: we first compute the local tangents and bitangents in order to take into account the local anisotropy. We infer most of the parameters for the computation of the $S_{d}$ function from known specifications of CDs: we set the distance between the slits to $\Delta_{x}=2.0 \mu \mathrm{m}$ and the size of the slits to $w_{x}=0.5 \mu \mathrm{m}$. Finally, we infer the only unknown parameter of the number of illuminated slits $\left(N_{x}=21\right)$ from our bokeh measurement of the CD (Fig. 9). We additionally modulate the 1D diffraction function $S_{d}$ with an anisotropic Gaussian windowing function in order to soften the edges of the pattern in the rendering. Fig. 21 compares the rendering of a $\mathrm{CD}$ under a point light source against a photograph. Here, the rendering is compared to the photograph of the $\mathrm{CD}$ in the presence of some ambient room illumination in order to make the $\mathrm{CD}$ itself visible (reflecting the room and camera) besides the colored diffraction pattern. As can be seen, the rendering is a close match to the photograph in this case with the infered parameters. We believe the bokeh photography technique could be used in this manner to infer rendering parameters for other types of sharply specular diffractive materials.

\subsection{Rendering Complex Light Sources}

We finally present another application of our proposed data-driven rendering method where we demonstrate rendering results under more complex light sources including environmental illumination. 


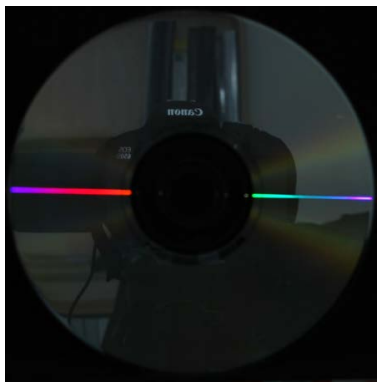

(a)

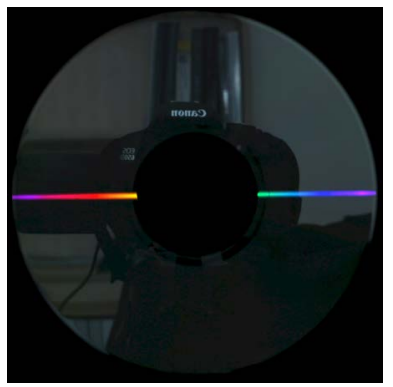

(b)

Fig. 21. Diffraction pattern on a CD due to a point light source. (a) Photograph. (b) Rendering.

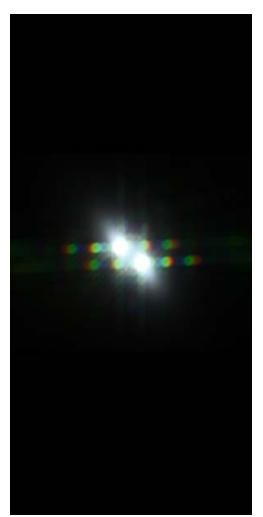

(a)

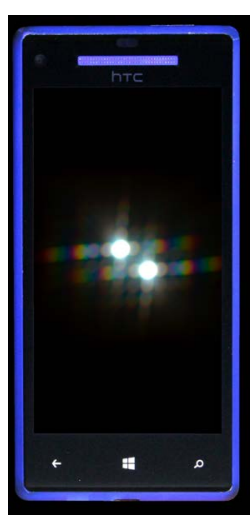

(b)
Fig. 22. Diffraction pattern due to a more complex light source (Philips Color Kinetics iW MR3). (a) Photograph. (b) Rendering using pre-convolved lookup table.

Fig. 22 demonstrates a rendering result for the HTC phone lit by a more complex LED light source (Philips Color Kinetics iW MR3) with two LEDs switched on which causes a superposition of two off-set diffraction patterns to appear in the photograph. We simulate this effect in rendering by convolving the measured diffraction of the HTC phone under a single point source with the point spread function (PSF) of the iW MR3 light source. We extracted the PSF of the light source from the photograph of the specular reflection of the light on the phone screen. Here, convolving the $S_{d}$ lookup table with the extracted PSF of the two LEDs gave better rendering results than pre-convolving the originally measured narrow band diffraction pattern (irradiance function $I$ ) before computing $S_{d}$. This is due to the fact the lookup table and the measured PSF contain RGB data whereas the irradiance pattern $I$ consists of a single spectral measurement. As can be seen, our pre-convolved diffraction lookup table is able to produce the two observed off-set diffraction patterns due to the two LEDs while still requiring storage of a single lookup table for real time rendering.
Fig. 2 (a) presents a rendering of the HTC phone in the Grace Cathedral environment [Debevec 2001]. Even when lit with environmental illumination, strong diffraction effects are visible on the phone screen due to the high frequency point lights in the Grace Cathedral. Interestingly, subtle diffraction effects can be seen even around the broader area lights reflected on the phone screen, such as the circular window in the center. Here, we assumed a full spectral power distribution of the environmental illumination for the $S_{d}$ lookup table of the diffraction pattern and converted that into sRGB color space for rendering. Additionally, for efficient real-time rendering, we pre-convolved each color channel of the Grace environment with data in the corresponding color channel of the RGB diffraction lookup table. Here, we assume normal incidence and employ the half-vector to index the look-up table and the environment map for the pre-filtering similar to the procedure described in [McAuley et al. 2013]. Finally, we employ the pre-filtered environment as a reflection map for real-time rendering of the resulting diffraction on the phone screen. Fig. 23 presents additional examples of rendering of the HTC and LG phone screens and a rough specular holographic paper in two different high frequency lighting environments. Please also see the accompanying video for more extensive visualization of diffraction under environmental illumination. Our renderings demonstrate that characteristic diffraction patterns can also be seen under environmental illumination with high frequency lighting. Finally, Fig. 24 presents a comparison of our employed pre-convolution approach for real time rendering to an offline reference rendering with full hemispherical integration. As can be seen, our pre-convolved diffraction rendering can very closely approximate the reference rendering result. In this case, we measured an RMSE error of $7.6 \%$ for the pre-convolved rendering compared to the offline rendering of the phone's LCD screen.

\section{DISCUSSION AND LIMITATIONS}

Our proposed data-driven approach can accurately and efficiently render diffraction effects on many common smooth planar surfaces. However, since the method relies on direct measurement of the diffraction pattern on the surface, it is not as well suited for finely textured or spatially varying diffractive surfaces such as various kinds of holographic papers with printed patterns (e.g., Fig. 25 a, b). In such materials, it can become difficult to automatically separate the observed diffraction pattern from the surface texture/pattern for building the $S_{d}$ lookup table. Our method currently also does not model diffraction effects in layered anisotropic holographic materials. A common example of this is called kinematic movement where the observed diffraction moves and changes with rotation around the sample (Fig. 25, c). Given that we rely on measurements with an LED flash as the light source, the illumination beam has a certain spatial spread on the sample. This might make it difficult to correctly measure the diffraction pattern on a spatially varying sample with very small disconnected diffractive regions as the specular highlight incident on the sample may be wider than a single unique diffractive patch. Some of these problems of measurement of spatially varying samples can be alleviated by employing an alternate setup commonly used in optics experiments [Ersoy 2006], where the diffraction pattern is not directly observed on the sample but it's reflection is observed on a screen. And smaller diffractive patches 


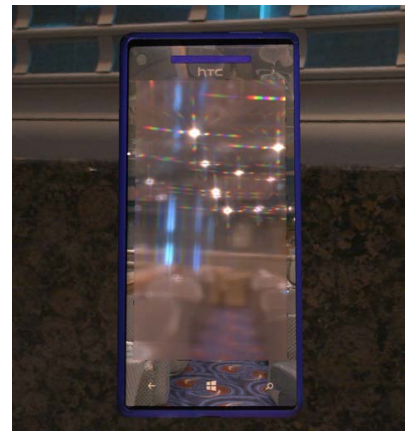

(a)

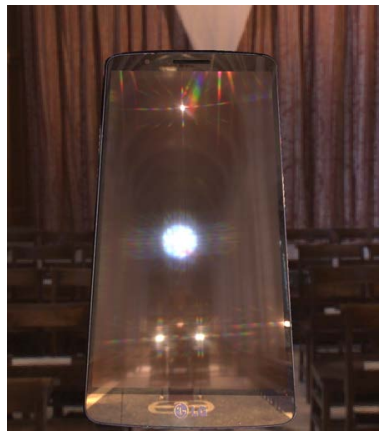

(b)

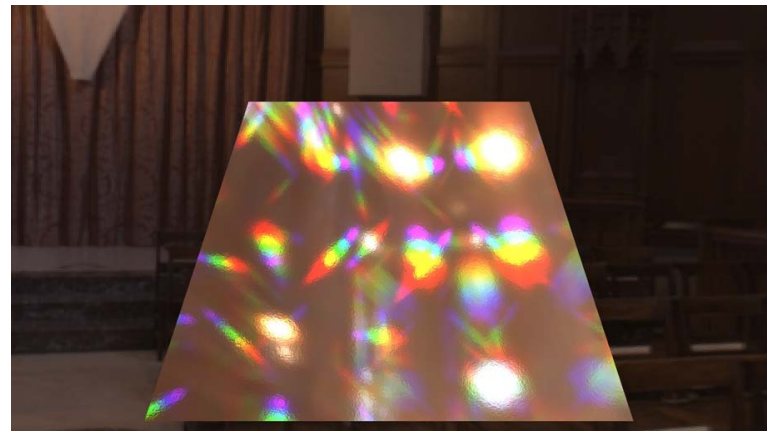

(c)

Fig. 23. Additional renderings under complex environmental illumination. (a) HTC phone. (b) LG phone. (c) Holographic paper. Here, reflections of spot lights in the interior environments exhibit characteristic diffraction patterns.

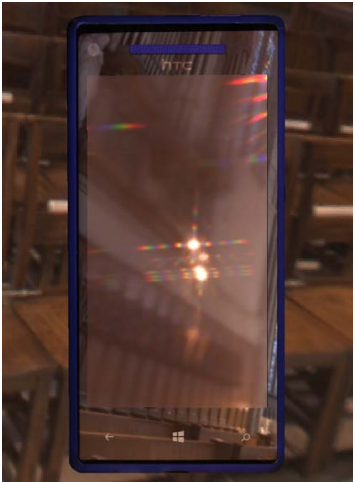

(a)

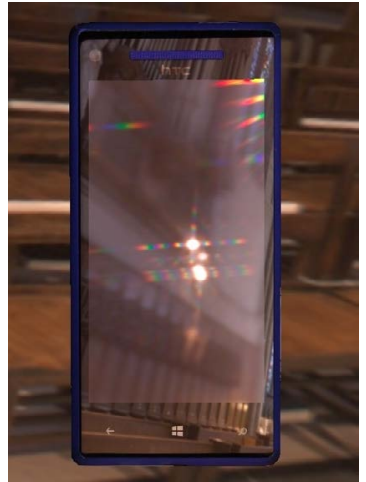

(b)

Fig. 24. Rendering comparison in the Grace Cathedral environment with (a) pre-convolution for real-time rendering, versus (b) reference offline rendering with full hemispherical integration.

can also be measured by employing a more collimated beam such as a laser source. However, diffractive materials are highly specular in general and employing a laser like source to image reflections off such samples can lead to eye safety issues. Hence, such measurements are more restrictive and best carried out in a laboratory setting.

The employed paraxial approximation for indexing the $S_{d}$ lookup table works well for rendering with distant light sources. However, it may result in visible errors when rendering with a very local light source (near-field) subtending large angles over the sample surface. Our pre-filtered rendering technique for environmental illumination assumes axis-aligned rendering of the diffractive sample and does not model off-axis rotation of the sample about the view vector (which should cause the diffraction pattern to rotate with the sample in the rendering). Our renderings also do not include depth-of-field effects which would be required in practice for accurately rendering such patterns on diffractive materials. This depth-of-field effect is noticeable in photographs where a sharp focus on the diffraction pattern leads to the sample itself being recorded slightly out-of-focus

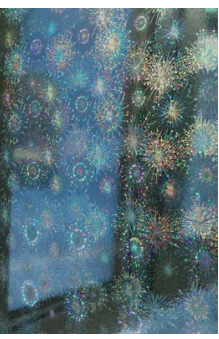

(a)

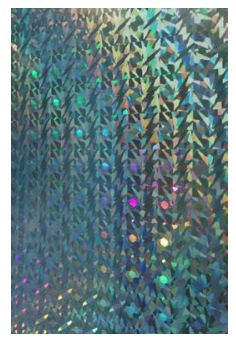

(b)

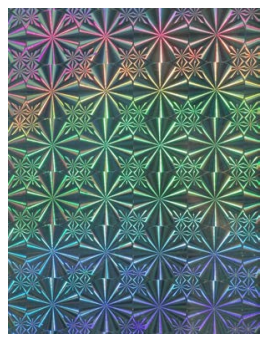

(c)
Fig. 25. Complex diffractive materials not modeled by our approach. (a) Holographic paper with fine texture pattern. (b) Spatially varying sample. (c) Gift bag exhibiting kinematic movement.

and vice-versa. This can however be easily added as an additional post-processing step.

Besides proposing rendering techniques for observed diffraction patterns, we also proposed a practical technique for measurement of diffraction gratings of highly specular samples using bokeh photography with an entry level DSLR camera and zoom lens. While our measurements can qualitatively produce diffraction effects when employed as gratings in a diffraction shader, we note that these measurements have limited spatial resolution (due to the employed commodity photography equipment) compared to high fidelity measurements with specialized microscopy equipment. We believe that better results can be obtained with our technique in practice by employing a professional grade DSLR camera and zoom lens with higher quality optics which should enable imaging of diffraction gratings at higher spatial resolution. Finally, both our proposed approaches of data-driven rendering and bokeh photography for grating measurement rely on a first order approximation of diffraction. While this works well in practice for diffraction due to periodic structures common in manufactured materials, it is unlikely to be sufficient for modeling diffraction due to irregular structures more commonly found in natural materials. Modeling such natural structures have been shown to require higher order representations [Dhillon et al. 2014; Dhillon and Ghosh 2016] which are somewhat incompatible with our first order measurements. 


\section{CONCLUSION}

To summarize, we have presented practical measurement based approaches for rendering diffraction effects in surface reflectance of homogeneous planar material samples. Such diffraction effects are becoming more common in everyday materials due to advances in manufacturing and fabrication techniques. We make the observation that while many such observed diffraction patterns are very complex to physically simulate using wave optics principles, they can be easily photographed in many cases. This inspires us to propose our practical data-driven approach for rendering complex diffraction patterns. Our method requires the precomputation of a single lookup table in a fragment shader which leads to a very practical solution for real time rendering applications such as games and VR Additionally, we present a practical approach for measurement of diffraction gratings of highly specular samples using regular photography equipment and show how to drive a diffraction shader using such measurements. Both our proposed approaches rely on a first order approximation which we find to be sufficient for rendering diffraction due to periodic structures in many common materials. The first order approximations also make the approaches very efficient for real-time rendering, including rendering under complex light sources and environmental illumination. We also demonstrate how our measurements can be useful for estimating parameters of analytic multislit diffraction for a few special cases which can be effectively rendered with such analytic solution.

In future work, it would be interesting to extend our approach for rendering more complex diffraction effects such as layered effects in a hologram or kinematic effects, as well as rendering highly spatially varying samples. We would also like to apply such a practical measurement based rendering approach for other iridescent effects such as thin film interference or dispersion effects in surface reflectance as well as iridescence in various scattering phenomena.

\section{ACKNOWLEDGEMENTS}

We thank Jérémy Riviere for assistance with data acquisition and the authors of [Dhillon et al. 2014] for providing an implementation of their diffraction shader. We also thank annonymous reviewers for their valuable inputs and Pieter Peers for feedback on an initial draft. Mirri provided us with samples of holographic papers. This work was supported by an EPSRC Early Career Fellowship EP/N006259/1 and a Royal Society Wolfson Research Merit Award. We also gratefully acknowledge the support of EPSRC Centre for Doctoral Training in High Performance Embedded and Distributed Systems (HiPEDS, Grant Reference EP/L016796/1).

\section{REFERENCES}

Emmanuel Agu and Francis S. Hill Jr. 2002. Diffraction Shading Models For Iridescent Surfaces. In Proc. IASTED VIIP.

R. L. Cook and K. E. Torrance. 1982. A Reflectance Model for Computer Graphics. ACM Trans. Graph. 1, 1 (Jan. 1982), 7-24. https://doi.org/10.1145/357290.357293

J.M. Cowley. 1995. Diffraction Physics. Elsevier Science. https://books.google.co.uk/ books?id=S3ekKcs2QoIC

Tom Cuypers, Tom Haber, Philippe Bekaert, Se Baek Oh, and Ramesh Raskar. 2012. Reflectance Model for Diffraction. ACM Trans. Graph. 31, 5, Article 122 (Sept. 2012), 11 pages. https://doi.org/10.1145/2231816.2231820

Paul Debevec. 2001. High-Resolution Light Probe Image Gallery. http://gl.ict.usc.edu/ Data/HighResProbes/. (2001).

Paul E. Debevec and Jitendra Malik. 1997. Recovering High Dynamic Range Radiance Maps from Photographs. In Proceedings of the 24th Annual Conference on Computer
Graphics and Interactive Techniques (SIGGRAPH '97). ACM Press/Addison-Wesley Publishing Co., New York, NY, USA, 369-378. https://doi.org/10.1145/258734.258884

D.S. Dhillon, J. Teyssier, M. Single, I. Gaponenko, M.C. Milinkovitch, and M. Zwicker 2014. Interactive Diffraction from Biological Nanostructures. Comput. Graph. Forum 33, 8 (Dec. 2014), 177-188. https://doi.org/10.1111/cgf.12425

Daljit Singh J. Dhillon and Abhijeet Ghosh. 2016. Efficient Surface Diffraction Renderings with Chebyshev Approximations. In SIGGRAPH ASIA 2016 Technical Briefs (SA '16). ACM, New York, NY, USA, Article 7, 4 pages. https://doi.org/10.1145/3005358. 3005376

Zhao Dong, Bruce Walter, Steve Marschner, and Donald P. Greenberg. 2015. Predicting Appearance from Measured Microgeometry of Metal Surfaces. ACM Trans. Graph. 35, 1, Article 9 (Dec. 2015), 13 pages. https://doi.org/10.1145/2815618

Edmund Optics. 2016. Diffraction Limit. (2016). http://www.edmundoptics. com/resources/application-notes/imaging/diffraction-limit/ Online; accessed 10November-2016.

O.K. Ersoy. 2006. Diffraction, Fourier Optics and Imaging. Wiley. https://books.google. co.uk/books?id=hpyUlD4OEdsC

Jeppe Revall Frisvad, Niels Jørgen Christensen, and Henrik Wann Jensen. 2007. Computing the Scattering Properties of Participating Media Using Lorenz-Mie Theory. ACM Trans. Graph. 26, 3, Article 60 (July 2007). https://doi.org/10.1145/1276377.1276452

Abhijeet Ghosh, Tongbo Chen, Pieter Peers, Cyrus A. Wilson, and Paul Debevec 2009. Estimating Specular Roughness and Anisotropy from Second Order Spherical Gradient Illumination. Computer Graphics Forum 28, 4 (2009), 1161-1170. https://doi.org/10.1111/j.1467-8659.2009.01493.x

Xavier Granier and Wolfgang Heidrich. 2003. A Simple Layered RGB BRDF Model Graph. Models 65, 4 (July 2003), 171-184. https://doi.org/10.1016/S1524-0703(03) 00042-0

Xiao D. He, Kenneth E. Torrance, François X. Sillion, and Donald P. Greenberg. 1991. A comprehensive physical model for light reflection. SIGGRAPH Comput. Graph. 25, 4 (1991), 175-186.

Eugene Hecht. 2014. Optics. Harlow : Pearson Education Limited.

Matthias B. Hullin, Elmar Eisemann, Hans-Peter Seidel, and Sungkil Lee. 2011 Physically-Based Real-Time Lens Flare Rendering. ACM Trans. Graph. (Proc. SIGGRAPH 2011) 30, 4 (2011), 108:1-108:9.

Matthias B. Hullin, Johannes Hanika, Boris Ajdin, Hans-Peter Seidel, Jan Kautz, and Hendrik P. A. Lensch. 2010. Acquisition and Analysis of Bispectral Bidirectional Reflectance and Reradiation Distribution Functions. ACM Trans. Graph. 29, 4, Article 97 (July 2010), 7 pages. https://doi.org/10.1145/1778765.1778834

Masataka Imura, Osamu Oshiro, Masahiko Saeki, Yoshitsugu Manabe, Kunihiro Chihara, and Yoshihiro Yasumuro. 2009. A Generic Real-time Rendering Approach for Structural Colors. In Proceedings of the 16th ACM Symposium on Virtual Reality Software and Technology (VRST '09). ACM, New York, NY, USA, 95-102. https://doi.org/10.1145/1643928.1643952

Young-Min Kang, Do-Hoon Lee, and Hwan-Gue Cho. 2015. Multipeak Anisotropic Microfacet Model for Iridescent Surfaces. Multimedia Tools Appl. 74, 16 (Aug. 2015), 6229-6242. https://doi.org/10.1007/s11042-014-2092-1

Anat Levin, Daniel Glasner, Ying Xiong, Frédo Durand, William Freeman, Wojciech Matusik, and Todd Zickler. 2013. Fabricating BRDFs at High Spatial Resolution Using Wave Optics. ACM Trans. Graph. 32, 4, Article 144 (July 2013), 14 pages. https://doi.org/10.1145/2461912.2461981

Marc Levoy, Ren Ng, Andrew Adams, Matthew Footer, and Mark Horowitz. 2006. Light Field Microscopy. ACM Trans. Graph. 25, 3 (July 2006), 924-934.

Clifford Lindsay and Emmanuel Agu. 2006. Physically-based Real-time Diffraction Using Spherical Harmonics. In Proceedings of the Second International Conference on Advances in Visual Computing - Volume Part I (ISVC'06). Springer-Verlag, Berlin, Heidelberg, 505-517. https://doi.org/10.1007/11919476_51

Stephen R. Marschner, Stephen H. Westin, Eric P. F. Lafortune, Kenneth E. Torrance, and Donald P. Greenberg. 1999. Image-based BRDF Measurement Including Human Skin. In Rendering Techniques.

Heylal Mashaal, Alex Goldstein, Daniel Feuermann, and Jeffrey M. Gordon. 2012. First direct measurement of the spatial coherence of sunlight. Optics Letters 37, 17 (2012), 3516-3518.

Wojciech Matusik, Hanspeter Pfister, Matt Brand, and Leonard McMillan. 2003. A data-driven reflectance model. In ACM TOG. 759-769.

Stephen McAuley, Stephen Hill, Adam Martinez, Ryusuke Villemin, Matt Pettineo, Dimitar Lazarov, David Neubelt, Brian Karis, Christophe Hery, Naty Hoffman, and Hakan Zap Andersson. 2013. Physically Based Shading in Theory and Practice. In ACM SIGGRAPH 2013 Courses (SIGGRAPH '13). ACM, New York, NY, USA, Article 22, 8 pages. https://doi.org/10.1145/2504435.2504457

Ankit Mohan, Grace Woo, Shinsaku Hiura, Quinn Smithwick, and Ramesh Raskar. 2009. Bokode: Imperceptible Visual Tags for Camera Based Interaction from a Distance. ACM Trans. Graph. 28, 3, Article 98 (July 2009), 8 pages. https://doi.org/10.1145/ 1531326.1531404

A. Musbach, G. W. Meyer, F. Reitich, and S. H. Oh. 2013. Full Wave Modelling of Light Propagation and Reflection. Comput. Graph. Forum 32, 6 (Sept. 2013), 24-37. https://doi.org/10.1111/cgf.12012 
Addy Ngan, Frédo Durand, and Wojciech Matusik. 2005. Experimental Analysis of BRDF Models. In Rendering Techniques. 117-226.

Se Baek Oh, Sriram Kashyap, Rohit Garg, Sharat Chandran, and Ramesh Raskar. 2010 Rendering Wave Effects with Augmented Light Field. Computer Graphics Forum 29, 2 (2010), 507-516. https://doi.org/10.1111/j.1467-8659.2009.01620.x

Iman Sadeghi, Adolfo Munoz, Philip Laven, Wojciech Jarosz, Francisco Seron, Diego Gutierrez, and Henrik Wann Jensen. 2012. Physically-based Simulation of Rainbows. ACM Trans. Graph. 31, 1, Article 3 (Feb. 2012), 12 pages. https://doi.org/10.1145/ 2077341.2077344

Jos Stam. 1999. Diffraction Shaders. In Proceedings of the 26th Annual Conference on Computer Graphics and Interactive Techniques (SIGGRAPH '99). ACM Press/AddisonWesley Publishing Co., New York, NY, USA, 101-110. https://doi.org/10.1145/311535 311546

Henry Stark. 2012. Application of optical fourier transforms. Elsevier

Yinlong Sun. 2000. A Spectrum-based Framework for Realistic Image Synthesis. Ph.D. Dissertation. Advisor(s) Fracchia, F. David. AAINQ61686.

Yinlong Sun. 2006. Rendering Biological Iridescences with RGB-based Renderers. ACM Trans. Graph. 25, 1 (Jan. 2006), 100-129. https://doi.org/10.1145/1122501.1122506

Yinlong Sun, Mark S. Drew, and F. David Fracchia. 1999a. Representing Spectral Functions by a Composite Model of Smooth and Spiky Components for Efficient Full-Spectrum Photorealism. In Proceedings of the 1999 IEEE Workshop on Photometric Modeling for Computer Vision and Graphics (PMCVG '99). IEEE Computer Society, Washington, DC, USA, 4-. http://dl.acm.org/citation.cfm?id=519626.826724

Yinlong Sun, F. David Fracchia, Thomas W. Calvert, and Mark S. Drew. 1999b. Deriving Spectra from Colors and Rendering Light Interference. IEEE Comput. Graph. Appl. 19, 4 (July 1999), 61-67. https://doi.org/10.1109/38.773965

Yinlong Sun, F. David Fracchia, Mark S. Drew, and Thomas W. Calvert. 2000. Rendering Iridescent Colors of Optical Disks. In Proceedings of the Eurographics Workshop on Rendering Techniques 2000. Springer-Verlag, London, UK, UK, 341-352. http: //dl.acm.org/citation.cfm?id=647652.732138

ThorLabs. 2015. Visible Ruled Reflective Diffraction Gratings. https://www.thorlabs.de/ newgrouppage9.cfm?objectgroup id=8626. (2015). Online; accessed 20-August-2015.

Nicolas Tsingos, Thomas Funkhouser, Addy Ngan, and Ingrid Carlbom. 2001. Modeling Acoustics in Virtual Environments Using the Uniform Theory of Diffraction. In Proceedings of the 28th Annual Conference on Computer Graphics and Interactive Techniques (SIGGRAPH '01). ACM, New York, NY, USA, 545-552. https://doi.org/10. $1145 / 383259.383323$

Chun-Po Wang, Noah Snavely, and Steve Marschner. 2011. Estimating Dual-scale Properties of Glossy Surfaces from Step-edge Lighting. ACM Trans. Graph. 30, 6, Article 172 (Dec. 2011), 12 pages. https://doi.org/10.1145/2070781.2024206

Jiaping Wang, Shuang Zhao, Xin Tong, John Snyder, and Baining Guo. 2008. Modeling Anisotropic Surface Reflectance with Example-based Microfacet Synthesis. ACM Trans. Graph. 27, 3, Article 41 (Aug. 2008), 9 pages. https://doi.org/10.1145/1360612. 1360640

Gregory J. Ward. 1992. Measuring and Modeling Anisotropic Reflection. SIGGRAPH Comput. Graph. 26, 2 (July 1992), 265-272. https://doi.org/10.1145/142920.134078

T. Wilson, M.A.A. Neil, and R. Juskaitis. 1998. Real-time three-dimensional imaging of macroscopic structures. Fournal of Microscopy 191, 2 (1998), 116-118.

Fu-Kun Wu and Chang-Wen Zheng. 2013. A Comprehensive Geometrical Optics Application for Wave Rendering. Graph. Models 75, 6 (Nov. 2013), 318-327. https //doi.org/10.1016/j.gmod.2013.07.004

Genzhi Ye, Sundeep Jolly, V. Micheal Bove, Qionghai Dai, Ramesh Raskar, and Gordon Wetzstein. 2014. Toward BxDF Display using Multilayer Diffraction. ACM Trans. Graph. (SIGGRAPH Asia) 33, 6 (2014).

Remo Ziegler, Simone Croci, and Markus Gross. 2008. Lighting and Occlusion in a Wave-Based Framework. Computer Graphics Forum 27, 2 (2008), 211-220. https //doi.org/10.1111/j.1467-8659.2008.01118.x

\section{A OPTICAL PATH APPROXIMATION}

The optical path in the Huygens-Fresnel integral (Equation 1) can be calculated as $\delta_{S P M}=\delta_{S P}+\delta_{P M}$ assuming the air as a medium. The optical path $\delta_{S P}=\delta_{S A}$ (see Fig. 26). Therefore its value can be calculated as follows :

$$
\delta_{S P}=\delta_{S A}=\delta_{S O}-O P \sin (\alpha)=\delta_{S O}+\overrightarrow{O P} \cdot \vec{u}_{S}
$$

The optical path $\delta_{P M}$ can be expressed as :

$$
\delta_{P M}=P M=\sqrt{(u-x)^{2}+(v-y)^{2}+L^{2}}
$$

A first order Taylor expansion of $\frac{\delta_{P M}}{\lambda}$ in the integral then gives:

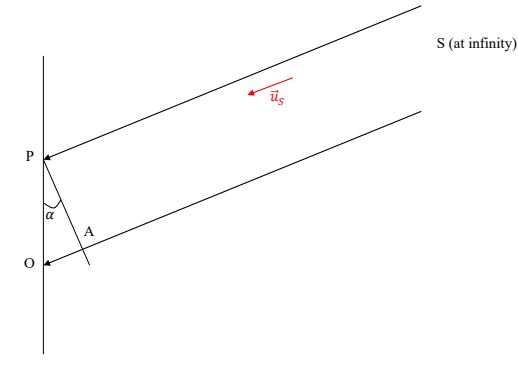

Fig. 26. Difference in optical path for between a ray from $\mathrm{S}$ to $\mathrm{P}$ and a ray from $\mathrm{S}$ to $\mathrm{O}$

$$
\frac{\delta_{P M}}{\lambda}=\frac{P M}{\lambda} \approx \frac{L}{\lambda}\left(1+\frac{(u-x)^{2}+(v-y)^{2}}{2 L^{2}}\right)
$$

This can be expanded in this form :

$$
\frac{\delta_{P M}}{\lambda}=\frac{L}{\lambda}-\frac{x u+v y}{L \lambda}+\frac{\left(x^{2}+u^{2}\right)+\left(y^{2}+v^{2}\right)}{2 L \lambda}
$$

Ignoring the second order terms in the above expansion in the Fraunhofer condition of diffraction (negligible magnitude) leads to a first order approximation of Eq. 1:

$a(M, \lambda)=\frac{i s_{o}}{\lambda L} e^{i\left(\omega t+i k\left(\delta_{S O}+L\right)\right)} \iint_{\Sigma} h(x, y) e^{-i k\left(\frac{x u+v y}{L}-\overrightarrow{O P} \cdot \vec{u}_{S}\right)} d x d y$

\section{B FIRST ORDER DIFFRACTION MODEL}

We employ a simplified diffraction model in this work that is related to Stam's BRDF [1999] through a first order (Born) approximation. Stam's BRDF is given by Eq. 22:

$$
f_{r, \text { diffraction }}\left(\vec{\omega}_{i}, \vec{\omega}_{o}, \lambda\right)=\frac{k^{2} F^{2}}{4 \pi^{2}} \frac{G}{A w^{2}}<|P(k u, k v)|^{2}>
$$

Here, the term $P(k u, k v)$ is given by the Fourier transform of the $p(x, y)=e^{i k w h(x, y)}$ function.

$$
P(k u, k v)=\iint_{\Sigma} p(x, y) e^{-\frac{2 i \pi}{\lambda}(x u+y v)} d x d y
$$

With a first order approximation, the $p(x, y)$ function in Stam's model becomes $p(x, y)=1+i k w h(x, y)$. Here, the 1 factor contributes to the specular reflection and the $i k w h$ term contributes to the diffraction.

$$
\begin{gathered}
P(k u, k v)=\iint_{\Sigma}(1+i w k h(x, y)) e^{-\frac{2 i \pi}{\lambda}(x u+y v)} d x d y \\
P(k u, k v)=\delta(k u, k v)+i w k H(k u, k v)
\end{gathered}
$$

where $H$ is the Fourier transform of the height field $h$ and $\delta$ the delta Dirac function. Hence, with the first order approximation, the diffractive component of Stam's BRDF can be written as:

$$
f_{r, \text { diffraction }}\left(\vec{\omega}_{i}, \vec{\omega}_{o}, \lambda\right) \propto \frac{4 \pi^{2} F^{2} G}{\lambda^{4}}<|H(k u, k v)|^{2}>
$$


The coefficient of proportionality in the above equation is $\frac{1}{A}$. Note that the microstructure of the diffractive sample is assumed to be homogeneous. Hence a change in the area of integration changes the value of $\left\langle|H(k u, k v)|^{2}>\right.$ proportionally to $A$ so that the ratio $\frac{\left\langle|H(k u, k v)|^{2}\right\rangle}{A}$ remains the same. As a result $\frac{1}{A}$ only modifies the brightness of the diffraction pattern. This is taken care of in the rendering with an exposure setting which is why the derivations in section 4.2 omit the proportionality factor.

\section{BOKEH CALIBRATION}

We need to calibrate for the unknown spatial scale of observations of the diffraction grating using the bokeh method described in section 5. In order to do this, we used a known blazed diffraction grating [ThorLabs 2015] specified to have 600 lines per millimetre and observed it using the bokeh method. The diffraction grating seen through the bokeh is shown in Fig. 27. Note that the specular reflection is orange even when it is illuminated under white light due to the spectral reflectance profile of the grating which integrates to the orange part of the visible spectrum. As the diffraction grating has 600 lines per millimeter and we observe around 106 lines inside the bokeh, we can infer that a pixel inside the bokeh represents $0.083 \mu \mathrm{m}$. We also plot the average height field along several horizontal cross sections of the bokeh in Fig. 29 (using green channel data since there is no blue reflectance) and compare it to the specifications of the grating given in Fig. 28. The red plot of the height field shows a periodicity due to the lines of the grating but does not show a regular slope as described in the specifications. This is due to the blur in imaging setup (due to lens aperture and optics) with our commodity photography equipment. The orange plot shows the corresponding height field after deconvolution with a more prominent blaze profile. These two plots do not directly match the real height field given by the blue curve. As a result we solve the optimisation problem given in equation $27(N$ is the number of samples along the $\mathrm{x}$ axis) to find an affine transformation of the deconvolved grating profile that best match the real grating profile. The final result has a RMSE of $5.83 \%$ which gives us a quantification of the error produced by the diffraction limit of the lens. We then apply that same affine transformation to the measured gratings of the phones and CD (see section 5) before computing the Taylor series lookup tables for rendering according to [Dhillon et al. 2014].

$$
\arg \min _{a, b} \sum_{k=1}^{N}\left(h_{\text {real }, k}-a\left(h_{\text {deconvolved }, k}-b\right)\right)^{2}
$$

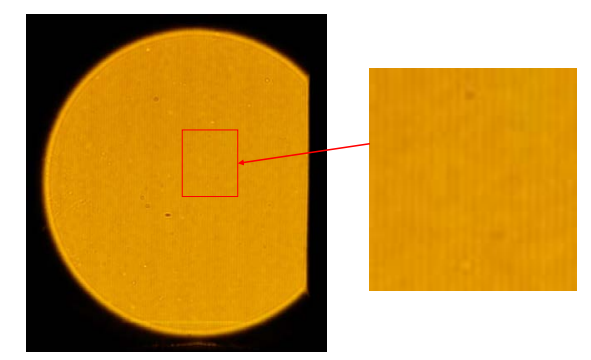

Fig. 27. (Left) ThorLabs diffraction grating seen through a bokeh. (Right) Zoom into smaller cross-section reveals the blazed lines.

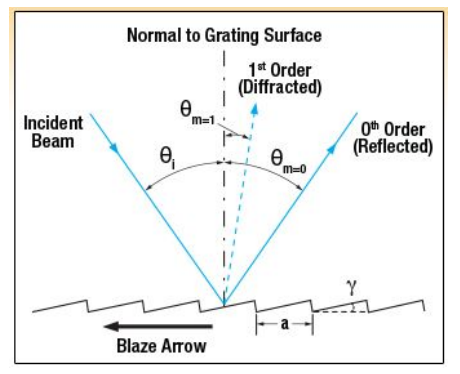

Fig. 28. Blazed diffraction grating diagram (reproduced with permission from [ThorLabs 2015]).
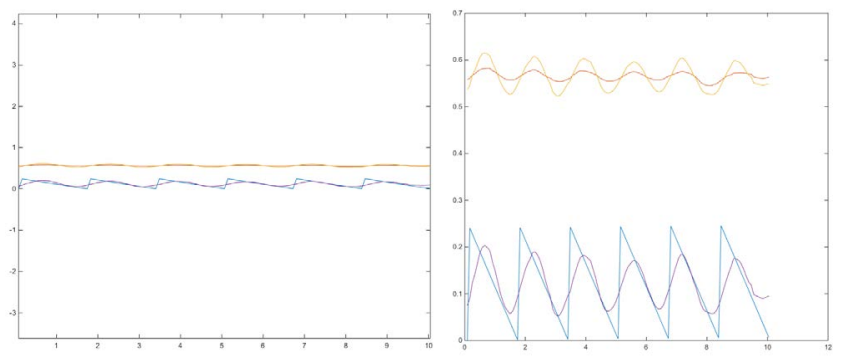

Fig. 29. Height field of the ThorLabs blazed diffraction grating measured along a horizontal cross-section (horizontal axis in micrometers). Red : Measured profile of intensity field affected by lens blur. Orange : Deconvolved blaze grating profile. Blue : True grating profile according to ThorLabs specification. Purple : Best affine transformation of the deconvolved height field. Left : True scale visualization. Right : Scaled y-axis for zoom. 